Discrete Comput Geom 36:205-265 (2006)

DOI: $10.1007 / \mathrm{s} 00454-005-1215-\mathrm{x}$

Geometry

\title{
Sphere Packings, VI. Tame Graphs and Linear Programs
}

\author{
Thomas C. Hales \\ Department of Mathematics, University of Pittsburgh, \\ Pittsburgh, PA 15217, USA \\ hales@pitt.edu
}

\begin{abstract}
This paper is the sixth and final part in a series of papers devoted to the proof of the Kepler conjecture, which asserts that no packing of congruent balls in three dimensions has density greater than the face-centered cubic packing. In a previous paper in this series, a continuous function $f$ on a compact space is defined, certain points in the domain are conjectured to give the global maxima, and the relation between this conjecture and the Kepler conjecture is established. In this paper we consider the set of all points in the domain for which the value of $f$ is at least the conjectured maximum. To each such point, we attach a planar graph. It is proved that each such graph must be isomorphic to a tame graph, of which there are only finitely many up to isomorphism. Linear programming methods are then used to eliminate all possibilities, except for three special cases treated in earlier papers: pentahedral prisms, the face-centered cubic packing, and the hexagonal-close packing. The results of this paper rely on long computer calculations.
\end{abstract}

\section{Introduction}

This paper is the last in the series of paper devoted to the proof of the Kepler conjecture. The first several sections prove a result that asserts that "all contravening graphs are tame." A contravening graph is one that is attached to a potential counterexample to the Kepler conjecture. Contravening graphs by nature are elusive and are studied by indirect methods. In contrast, the defining properties of tame graphs lend themselves to direct examination. (By definition, tame graphs are planar graphs such that the degree of every vertex is at least two and at most six, the length of every face is at least 3 and at most 8 , and such that other similar explicit properties hold true.)

It is no coincidence that contravening graphs all turn out to be tame. The definition of a tame graph has been tailored to suit the situation at hand. We set out to prove explicit properties of contravening graphs, and when we are satisfied with what we have proved, we brand a graph with these properties a tame graph. 
The first section of this paper gives the definition of a tame graph. The second section gives the classification of all tame graphs. There are several thousand such graphs. The classification was carried out by computer. This classification is one of the main uses of a computer in the proof of the Kepler conjecture. A detailed description of the algorithm that is used to find all tame graphs is presented in this section.

The third section of this paper gives a review of results from earlier parts of the paper that are relevant to the study of tame plane graphs. In the abridged version of the proof [Ha15], the results cited in this section are treated as axioms. This section thus serves as a guide to the results that are proved in this volume, but not in the abridged version of the proof.

This section also contains a careful definition of what it means to be a contravening plane graph. The first approximation to the definition is that it is the combinatorial plane graph associated with the net of edges on the unit sphere bounding the standard regions of a contravening decomposition star. The precise definition is somewhat more subtle because we wish ensure that every face of a contravening plane graph is a simple polygon. To guarantee that this property holds, we simplify the net of edges on the unit sphere whenever necessary.

The fourth and fifth sections of this paper contain the proof that all contravening plane graphs are tame. These sections complete the first half of this paper.

The second half of this paper is about linear programming. Linear programs are used to prove that with the exception of three tame graphs (those attached to the face-centered cubic packing, the hexagonal-close packing, and the pentahedral prism), a tame graph cannot be a contravening graph. This result reduces the proof of the Kepler conjecture to a close examination of three graphs. Pentahedral prism graphs are treated in the fifth paper. The face-centered cubic and hexagonal-close packing graphs are treated in Section 8 of the third paper. The linear programming results together with these earlier results complete the proof of the Kepler conjecture.

The sixth section of this paper describes how to attach a linear program to a tame plane graph. The output from this linear program is an upper bound on the score of all decomposition stars associated with the given tame plane graph. The seventh section of this paper shows how to use linear programs to eliminate what are called the aggregate tame plane graphs. The aggregates are those cases where the net of edges formed by the edges of standard regions was simplified to ensure that every face of a contravening plane graph is a polygon. By the end of this section, we have a proof that every standard region in a contravening decomposition star is bounded by a simple polygon.

The final section of this paper gives a long list of special strategies that are used when the output from the linear program in the sixth section does not give conclusive results. The general strategy is to partition the original linear program into a collection of refined linear programs with the property that the score is no greater than the maximum of the outputs from the linear programs in the collection. These branch and bound strategies are described in this final section. Linear programming shows that every decomposition star with a tame plane graph (other than the three mentioned above) has a score less than that of the decomposition stars attached to the face-centered cubic packing. This and earlier results imply the Kepler conjecture. 


\section{Tame Graphs}

This section defines a class of plane graphs. Graphs in this class are said to be tame. In the next section we give a complete classification of all tame graphs. This classification of tame graphs was carried out by computer and is a major step of the proof of the Kepler conjecture.

\subsection{Basic Definitions}

Definition 18.1. An $n$-cycle is a finite set $C$ of cardinality $n$, together with a cyclic permutation $s$ of $C$. We write $s$ in the form $v \mapsto s(v, C)$, for $v \in C$. The element $s(v, C)$ is called the successor of $v$ (in $C$ ). A cycle is an $n$-cycle for some natural number $n$. By abuse of language, we often identify $C$ with the cycle. The natural number $n$ is the length of the cycle.

Definition 18.2. Let $G$ be a nonempty finite set of cycles (called faces) of length at least 3 . The elements of faces are called the vertices of $G$. An unordered pair of vertices $\{v, w\}$ such that one element is the successor of the other in some face is called an edge. The vertices $v$ and $w$ are then said to be adjacent. The set $G$ is a plane graph if four conditions hold:

1. If an element $v$ has successor $w$ in some face $F$, then there is a unique face (call it $\left.s^{\prime}(F, v)\right)$ in $G$ for which $v$ is the successor of $w$. (Thus, $v=s\left(w, s^{\prime}(F, v)\right)$, and each edge occurs twice with opposite orientation.)

2. For each vertex $v$, the function $F \mapsto s^{\prime}(F, v)$ is a cyclic permutation of the set of faces containing $v$.

3. Euler's formula holds relating the number of vertices $V$, the number of edges $E$, and the number of faces $F$ :

$$
V-E+F=2 .
$$

4. The set of vertices is connected. That is, the only nonempty set of vertices that is closed under $v \mapsto s(v, C)$ for all $C$ is the full set of vertices.

Remark 18.3. The set of vertices and edges of a plane graph form a planar graph in the usual graph-theoretic sense of admitting an embedding into the plane. Every planar graph carries an orientation on its faces that is inherited from an orientation of the plane. (Use the right-hand rule on the face, to orient it with the given outward normal of the oriented plane.) For us, the orientation is built into the definition, so that properly speaking, we should call these objects oriented plane graphs. We follow the convention of distinguishing between planar graphs (which admit an embedding into the plane) and plane graphs (for which a choice of embedding has been made). Our definition is more restrictive than the standard definition of plane graph in the literature, because we require all faces to be simple polygons with at least three vertices. Thus, a graph with a single edge does not comply with our narrow definition of plane graph. Other graphs 

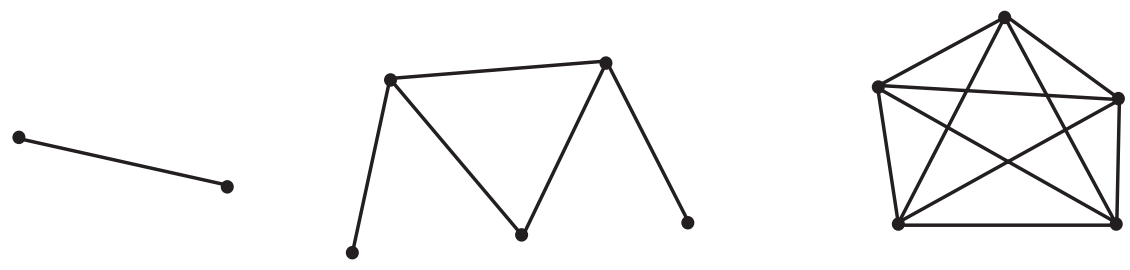

Fig. 18.1. Some examples of graphs that are excluded from the narrow definition of plane graph, as defined in this section.

that are excluded by this definition are shown in Fig. 18.1. Standard results about plane graphs can be found in any of a number of graph theory textbooks. However, this paper is written in such a way that it should not be necessary to consult outside graph theory references.

Definition 18.4. Let len be the length function on faces. Faces of length 3 are called triangles, those of length 4 are called quadrilaterals, and so forth. Let tri $(v)$ be the number of triangles containing a vertex $v$. A face of length at least 5 is called an exceptional face.

Two plane graphs are properly isomorphic if there is a bijection of vertices inducing a bijection of faces. For each plane graph, there is an opposite plane graph $G^{\mathrm{op}}$ obtained by reversing the cyclic order of vertices in each face. A plane graph $G$ is isomorphic to another if $G$ or $G^{\mathrm{op}}$ is properly isomorphic to the other.

Definition 18.5. The degree of a vertex is the number of faces it belongs to. An $n$ circuit in $G$ is a cycle $C$ in the vertex-set of $G$, such that for every $v \in C$, it forms an edge in $G$ with its successor: that is, $(v, s(v, C))$ is an edge of $G$.

In a plane graph $G$ we have a combinatorial form of the Jordan curve theorem: each $n$-circuit determines a partition of $G$ into two sets of faces.

Definition 18.6. The type of a vertex is defined to be a triple of nonnegative integers $(p, q, r)$, where $p$ is the number of triangles containing the vertex, $q$ is the number of quadrilaterals containing it, and $r$ is the number of exceptional faces. When $r=0$, we abbreviate the type to the ordered pair $(p, q)$.

\subsection{Weight Assignments}

We call the constant tgt $=14.8$, which arises repeatedly in this section, the target. (This constant arises as an approximation to $4 \pi \zeta-8 \approx 14.7947$, where $\zeta=$ $1 /(2 \arctan (\sqrt{2} / 5))$.) 
Define $a: \mathbb{N} \rightarrow \mathbb{R}$ by

$$
a(n)= \begin{cases}14.8, & n=0,1,2 \\ 1.4, & n=3 \\ 1.5, & n=4 \\ 0, & \text { otherwise }\end{cases}
$$

Define $b: \mathbb{N} \times \mathbb{N} \rightarrow \mathbb{R}$ by $b(p, q)=14.8$, except for the values in the following table (with tgt $=14.8$ ):

\begin{tabular}{cccccc}
\hline & \multicolumn{5}{c}{$q$} \\
\cline { 2 - 6 }$p$ & 0 & 1 & 2 & 3 & 4 \\
\hline 0 & tgt & tgt & tgt & 7.135 & 10.649 \\
1 & tgt & tgt & 6.95 & 7.135 & tgt \\
2 & tgt & 8.5 & 4.756 & 12.981 & tgt \\
3 & tgt & 3.642 & 8.334 & tgt & tgt \\
4 & 4.139 & 3.781 & tgt & tgt & tgt \\
5 & 0.55 & 11.22 & tgt & tgt & tgt \\
6 & 6.339 & tgt & tgt & tgt & tgt \\
\hline
\end{tabular}

Define $c: \mathbb{N} \rightarrow \mathbb{R}$ by

$$
c(n)= \begin{cases}1, & n=3, \\ 0, & n=4, \\ -1.03, & n=5, \\ -2.06, & n=6 \\ -3.03, & \text { otherwise }\end{cases}
$$

Define $d: \mathbb{N} \rightarrow \mathbb{R}$ by

$$
d(n)= \begin{cases}0, & n=3, \\ 2.378, & n=4, \\ 4.896, & n=5, \\ 7.414, & n=6, \\ 9.932, & n=7 \\ 10.916, & n=8 \\ \operatorname{tgt}=14.8, & \text { otherwise. }\end{cases}
$$

A set $V$ of vertices is called a separated set of vertices if the following four conditions hold:

1. For every vertex in $V$ there is an exceptional face containing it.

2. No two vertices in $V$ are adjacent.

3. No two vertices in $V$ lie on a common quadrilateral.

4. Each vertex in $V$ has degree 5.

A weight assignment of a plane graph $G$ is a function $w: G \rightarrow \mathbb{R}$ taking values in the set of nonnegative real numbers. A weight assignment is admissible if the following properties hold:

1. If the face $F$ has length $n$, then $w(F) \geq d(n)$. 
2. If $v$ has type $(p, q)$, then

$$
\sum_{F: v \in F} w(F) \geq b(p, q)
$$

3. Let $V$ be any set of vertices of type $(5,0)$. If the cardinality of $V$ is $k \leq 4$, then

$$
\sum_{F: V \cap F \neq \emptyset} w(F) \geq 0.55 k .
$$

4. Let $V$ be any separated set of vertices. Then

$$
\sum_{F: V \cap F \neq \emptyset}(w(F)-d(\operatorname{len}(F))) \geq \sum_{v \in V} a(\operatorname{tri}(v)) .
$$

The sum $\sum_{F} w(F)$ is called the total weight of $w$.

\subsection{Plane Graph Properties}

We say that a plane graph is tame if it satisfies the following conditions:

1. The length of each face is at least 3 and at most 8 .

2. Every 3-circuit is a face or the opposite of a face.

3. Every 4-circuit surrounds one of the cases illustrated in Fig. 18.2.

4. The degree of every vertex is at least 2 and at most 6 .

5. If a vertex is contained in an exceptional face, then the degree of the vertex is at most 5 .

6.

$$
\sum_{F} c(\operatorname{len}(F)) \geq 8
$$

7. There exists an admissible weight assignment of total weight less than the target, $\operatorname{tgt}=14.8$.

8. There are never two vertices of type $(4,0)$ that are adjacent to each other.


Fig. 18.2. Tame 4-circuits. 
It follows from the definitions that the abstract vertex-edge graph of $G$ has no loops or multiple joins. Also, by construction, every vertex lies in at least two faces. Property 6 implies that the graph has at least eight triangles.

Remark 18.7. We pause to review the strategy of the proof of the Kepler conjecture as described in Section 3.2. The decomposition stars that violate the main inequality $\sigma(D) \geq 8 p t$ are said to contravene. A plane graph is associated with each contravening decomposition star. These are the contravening plane graphs. The main object of this paper is to prove that the only two contravening graphs are $G_{\text {fcc }}$ and $G_{\mathrm{hcp}}$, the graphs associated with the face-centered cubic and hexagonal-close packings.

We have defined a set of plane graphs, called tame graphs. The next section will give a classification of tame plane graphs. (There are several thousand.) Section 20 gives a proof that all contravening plane graphs are tame. By the classification result, this reduces the possible contravening graphs to an explicit finite list. Case-by-case linear programming arguments will show that none of these tame plane graphs is a contravening graph (except $G_{\text {fcc }}$ and $G_{\mathrm{hcp}}$ ). Having eliminated all possible graphs, we arrive at the resolution of the Kepler conjecture.

\section{Classification of Tame Plane Graphs}

\subsection{Statement of the Theorem}

A list of several thousand plane graphs appears in [Ha16]. The following theorem is listed as one of the central claims in the proof in Section 3.3.

Theorem 19.1. Every tame plane graph is isomorphic to a plane graph in this list.

The results of this section are not needed except in the proof of Theorem 19.1.

Computers are used to generate a list of all tame plane graphs and to check them against the archive of tame plane graphs. We describe a finite state machine that produces all tame plane graphs. This machine is not particularly efficient, and so we also include a description of pruning strategies that prevent a combinatorial explosion of possibilities.

\subsection{Basic Definitions}

In order to describe how all tame plane graphs are generated, we need to introduce partial plane graphs that encode an incompletely generated tame graph. A partial plane graph is itself a graph, but marked in such a way to indicate that it is in a transitional state that will be used to generate further plane graphs.

Definition 19.2. A partial plane graph is a plane graph with additional data: every face is marked as "complete" or "incomplete." We call a face complete or incomplete according to the markings. We require the following condition:

- No two incomplete faces share an edge. 
Each unmarked plane graph is identified with the marked plane graph in which every face is complete. We represent a partial plane graph graphically by deleting one face (the face at infinity) and drawing the others and shading those that are complete.

A patch is a partial plane graph $P$ with two distinguished faces $F_{1}$ and $F_{2}$, such that the following hold:

- Every vertex of $P$ lies in $F_{1}$ or $F_{2}$.

- The face $F_{2}$ is the only complete face.

- $F_{1}$ and $F_{2}$ share an edge.

- Every vertex of $F_{2}$ that is not in $F_{1}$ has degree 2 .

$F_{1}$ and $F_{2}$ will be referred to as the distinguished incomplete and the distinguished complete faces, respectively.

Patches can be used to modify a partial plane graph as follows. Let $F$ be an incomplete face of length $n$ in a partial plane graph $G$. Let $P$ be a patch whose incomplete distinguished face $F_{1}$ has length $n$. Replace $P$ with a properly isomorphic patch $P^{\prime}$ in which the image of $F_{1}$ is equal to $F^{\mathrm{op}}$ and in which no other vertex of $P^{\prime}$ is a vertex of $G$. Then

$$
G^{\prime}=\left\{F^{\prime} \in G \cup P^{\prime}: F^{\prime} \neq F^{\mathrm{op}}, F^{\prime} \neq F\right\}
$$

is a partial plane graph. Intuitively, we cut away the faces $F$ and $F_{1}$ from their plane graphs, and glue the holes together along the boundary (Fig. 19.1). (It is immediate that the condition in the definition of partial plane graphs (Definition 19.2) is maintained by this process.) There are $n$ distinct proper ways of identifying $F_{1}$ with $F^{\mathrm{op}}$ in this construction, and we let $\varphi$ be this identification. The isomorphism class of $G^{\prime}$ is uniquely determined by the isomorphism class of $G$, the isomorphism class of $P$, and $\varphi$ (ranging over proper bijections $\varphi: F_{1} \mapsto F^{\text {op }}$ ).

\subsection{A Finite State Machine}

For a fixed $N$ we define a finite state machine as follows. The states of the finite state machine are isomorphism classes of partial plane graphs $G$ with at most $N$ vertices. The
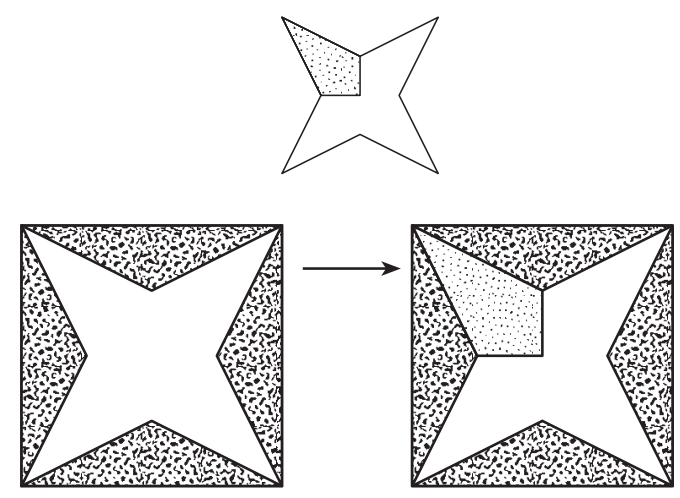

Fig. 19.1. Patching a plane graph. 
transitions from one state $G$ to another are isomorphism classes of pairs $(P, \varphi)$ where $P$ is a patch, and $\varphi$ pairs an incomplete face of $G$ with the distinguished incomplete face of $P$. However, we exclude a transition $(P, \varphi)$ at a state if the resulting partial plane graphs contains more than $N$ vertices. Figure 19.1 shows two states and a transition between them.

The initial states $I_{n}$ of the finite state machine are defined to be the isomorphism classes of partial plane graphs with two faces:

$$
\{(1,2, \ldots, n),(n, n-1, \ldots, 1)\},
$$

where $n \leq N$, one face is complete, and the other is incomplete. In other words, they are patches with exactly two faces.

A terminal state of this finite state machine is one in which every face is complete. By construction, these are (isomorphism classes of) plane graphs with at most $N$ vertices.

Lemma 19.3. Let $G$ be a plane graph with at most $N$ vertices. Then its state in the machine is reachable from an initial state through a series of transitions.

Proof. Pick a face in $G$ of length $n$ and identify it with the complete face in the initial state $I_{n}$. At any stage at state $G^{\prime}$, we have an identification of all of the vertices of the plane graph $G^{\prime}$ with some of the vertices of $G$, and an identification of all of the complete faces of $G^{\prime}$ with some of the faces of $G$ (all faces of $G$ are complete). Pick an incomplete face $F$ of $G^{\prime}$ and an oriented edge along that face. We let $F^{\prime}$ be the complete face of $G$ with that edge, with the same orientation on that edge as $F$. Create a patch with distinguished faces $F_{1}=F^{\mathrm{op}}$ and $F_{2}=F^{\prime}$. ( $F_{1}$ and $F_{2}$ determine the patch up to isomorphism.) It is immediate that the conditions defining a patch are fulfilled. Continue in this way until a graph isomorphic to $G$ is reached.

Remark 19.4. It is an elementary matter to generate all patches $P$ such that the distinguished faces have given lengths $n$ and $m$. Patching is also entirely algorithmic, and thus by following all paths through the finite state machine, we obtain all plane graphs with at most $N$ vertices.

\subsection{Pruning Strategies}

Although we reach all graphs in this manner, it is not computationally efficient. We introduce pruning strategies to increase the efficiency of the search. We can terminate our search along a path through the finite state machine, if we can determine one of the following:

1. Every terminal graph along that path violates one of the defining properties of tameness.

2. An isomorphic terminal graph will be reached by some other path that will not be terminated early. 
Here are some pruning strategies of type 1 above. They are immediate consequences of the conditions of the defining properties of tameness.

- If the current state contains an incomplete face of length 3, then eliminate all transitions, except for the transition that carries the partial plane graph to a partial plane graph that is the same in all respects, except that the face has become complete.

- If the current state contains an incomplete face of length 4, then eliminate all transitions except those that lead to the possibilities of Section 18.3, Property 3 , where in Property 3 each depicted face is interpreted as being complete.

- Remove all transitions with patches whose complete face has length greater than 8 .

- It is frequently possible to conclude from the examination of a partial plane graph that no matter what the terminal position, any admissible weight assignment will give total weight greater than the target $(\operatorname{tgt}=14.8)$. In such cases, all transitions out of the partial plane graph can be pruned.

To take a simple example of the last item, we observe that weights are always nonnegative, and that the weight of a complete face of length $n$ is at least $d(n)$. Thus, if there are complete faces $F_{1}, \ldots, F_{k}$ of lengths $n_{1}, \ldots, n_{k}$, then any admissible weight assignment has total weight at least $\sum_{i=1}^{k} d\left(n_{i}\right)$. If this number is at least the target, then no transitions out of that state need be considered.

More generally, we can apply all of the inequalities in the definition of admissible weight assignment to the complete portion of the partial plane graph to obtain lower bounds. However, we must be careful, in applying Property 4 of admissible weight assignments, because vertices that are not adjacent at an intermediate state may become adjacent in the complete graph. Also, vertices that do not lie together in a quadrilateral at an intermediate state may do so in the complete graph.

Here are some pruning strategies of type 2:

- At a given state it is enough to fix one incomplete face and one edge of that face and then to follow only the transitions that patch along that face and add a complete face along that edge. (This is seen from the proof of Lemma 19.3.)

- In leading out from the initial state $I_{n}$, it is enough to follow paths in which every added complete face has length at most $n$. (A graph with a face of length $m$, for $m>n$, will be also be found downstream from $I_{m}$.)

- Make a list of all type $(p, q)$ with $b(p, q)<\operatorname{tgt}=14.8$. Remove the initial states $I_{3}$ and $I_{4}$, and create new initial states $I_{p, q}\left(I_{p, q}^{\prime}, I_{p, q}^{\prime \prime}\right.$, etc. $)$ in the finite state machine. Define the state $I_{p, q}$ to be one consisting of $p+q+1$ faces, with $p$ complete triangles and $q$ complete quadrilaterals all meeting at a vertex (and one other incomplete face away from $v$ ). (If there is more than one way to arrange $p$ triangles and $q$ quadrilaterals, create states $I_{p, q}, I_{p, q}^{\prime}, I_{p, q}^{\prime \prime}$, for each possibility. See Fig. 19.2.) Put a linear order on states $I_{p, q}$. In state transitions downstream from $I_{p, q}$ disallow any transition that creates a vertex of type $\left(p^{\prime}, q^{\prime}\right)$, for any $\left(p^{\prime}, q^{\prime}\right)$ preceding $(p, q)$ in the imposed linear order.

This last pruning strategy is justified by the following lemma, which classifies vertices of type $(p, q)$. 

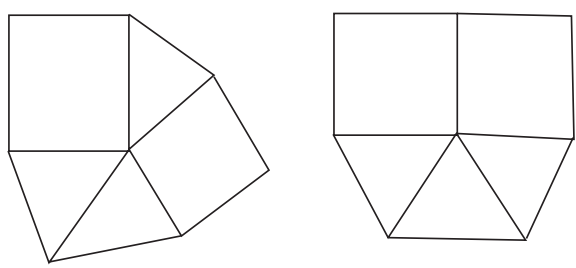

Fig. 19.2. States $I_{3,2}$ and $I_{3,2}^{\prime}$.

Lemma 19.5. Let $A$ and $B$ be triangular or quadrilateral faces that have at least two vertices in common in a tame graph. Then the faces have exactly two vertices in common, and an edge is shared by the two faces.

Proof. Exercise. Some of the configurations that must be ruled out are shown in Fig. 19.3. Some properties that are particularly useful for the exercise are Properties 2 and 3 of tameness, and Property 2 of admissibility.

Once a terminal position is reached it is checked to see whether it satisfies all the properties of tameness.

Duplication is removed among isomorphic terminal plane graphs. It is not an entirely trivial procedure for the computer to determine whether an isomorphism between two plane graphs exists. This is accomplished by computing a numerical invariant of a vertex that depends only on the local structure of the vertex. If two plane graphs are properly isomorphic then the numerical invariant is the same at vertices that correspond under the proper isomorphism. If two graphs have the same number of vertices with the same numerical invariants, they become candidates for an isomorphism. All possible numerical-invariant preserving bijections are attempted until a proper isomorphism is found, or until it is found that none exist. If there is no proper isomorphism, the same procedure is applied to the opposite plane graph to find any possible orientation-reversing isomorphism.

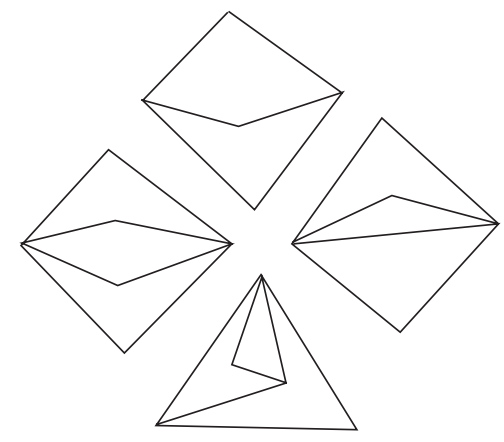

Fig. 19.3. Some impossibilities. 
This same isomorphism-producing algorithm is used to match each terminal graph with a graph in the archive. It is found that each terminal graph matches with one in the archive. (The archive was originally obtained by running the finite state machine and making a list of all the terminal states up to isomorphism that satisfy the given conditions.)

In this way Theorem 19.1 is proved.

\section{Contravening Graphs}

We have seen that a system of points and arcs on the unit sphere can be associated with a decomposition star $D$. The points are the radial projections of the vertices of $U(D)$ (those at distance at most $2 t_{0}=2.51$ from the origin). The arcs are the radial projections of edges between $v, w \in U(D)$, where $|v-w| \leq 2 t_{0}$. If we consider this collection of arcs combinatorially as a graph, then it is not always true that these arcs form a plane graph in the restrictive sense of Section 18.

The purpose of this section is to show that if the original decomposition star contravenes, then minor modifications can be made to the system of arcs of the graph so that the resulting combinatorial graph has the structure of a plane graph in the sense of Section 18 . These plane graphs are called contravening plane graphs, or simply contravening graphs.

\subsection{A Review of Earlier Results}

Let $\zeta=1 /(2 \arctan (\sqrt{2} / 5))$. Let $\operatorname{sol}(R)$ denote the solid angle of a standard region $R$. We write $\tau_{R}$ for the following modification of $\sigma_{R}$ :

$$
\tau_{R}(D)=\operatorname{sol}(R) \zeta p t-\sigma_{R}(D)
$$

and

$$
\tau(D)=\sum \tau_{R}(D)=4 \pi \zeta p t-\sigma(D) .
$$

Since $4 \pi \zeta p t$ is a constant, $\tau$ and $\sigma$ contain the same information, but $\tau$ is often more convenient to work with. A contravening decomposition star satisfies

$$
\tau(D) \leq 4 \pi \zeta p t-8 p t=(4 \pi \zeta-8) p t .
$$

The constant $(4 \pi \zeta-8) p t$ (and its upper bound tgt $p t$ where tgt $=14.8$ ) occurs repeatedly in the discussion that follows.

Recall that a standard cluster is a pair $(R, D)$ consisting of a decomposition $\operatorname{star} D$ and one of its standard regions $R$. If $F$ is a finite set (or finite union) of standard regions, let

$$
\sigma_{F}(D)=\sum_{R} \sigma_{R}(D), \quad \tau_{F}(D)=\sum_{R} \tau_{R}(D),
$$

where the sum runs over all the standard regions in $F$. When the sum runs over all standard regions,

$$
\sigma(D)=\sum \sigma_{R}(D), \quad \tau(D)=\sum \tau_{R}(D)
$$




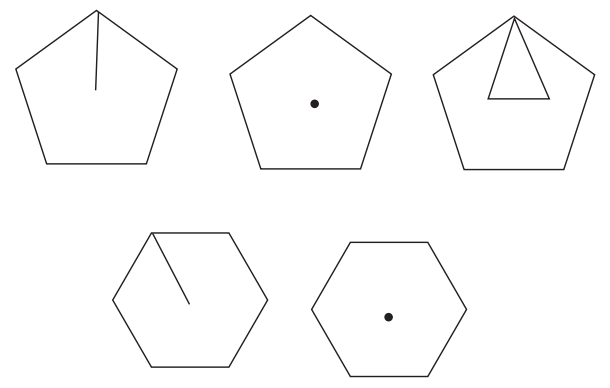

Fig. 20.1. Nonpolygonal standard regions $(n(R)=7,7,8,8,8)$.

A natural number $n(R)$ is associated with each standard region. If the boundary of that region is a simple polygon, then $n(R)$ is the number of sides. If the boundary consists of $k$ disjoint simple polygons, with $n_{1}, \ldots, n_{k}$ sides then

$$
n(R)=n_{1}+\cdots+n_{k}+2(k-1) .
$$

Lemma 20.1. Let $R$ be a standard region in a contravening decomposition star $D$. The boundary of $R$ is a simple polygon with at most eight edges, or one of the configurations of Fig. 20.1.

Proof. This is Theorem 12.1 and Corollary 12.2.

Lemma 20.2. Let $R$ be a standard region. We have $\tau_{R}(D) \geq t_{n}$, where $n=n(R)$, and

$$
\begin{gathered}
t_{3}=0, \quad t_{4}=0.1317, \quad t_{5}=0.27113, \\
t_{6}=0.41056, \quad t_{7}=0.54999, \quad t_{8}=0.6045 .
\end{gathered}
$$

Furthermore, $\sigma_{R}(D) \leq s_{n}$, for $5 \leq n \leq 8$, where

$$
\begin{gathered}
s_{3}=1 p t, \quad s_{4}=0, \quad s_{5}=-0.05704, \\
s_{6}=-0.11408, \quad s_{7}=-0.17112, \quad s_{8}=-0.22816 .
\end{gathered}
$$

Proof. This is Theorem 12.1.

Lemma 20.3. Let $F$ be a set of standard regions bounded by a simple polygon with at most nine edges. Assume that

$$
\sigma_{F}(D) \leq s_{9} \quad \text { and } \quad \tau_{F}(D) \geq t_{9},
$$

where $s_{9}=-0.1972$ and $t_{9}=0.6978$. Then $D$ does not contravene.

Proof. This is Section 12.2. 
Lemma 20.4. Let $(R, D)$ be a standard cluster. If $R$ is a triangular region, then

$$
\sigma_{R}(D) \leq 1 p t .
$$

If $R$ is not a triangular region, then

$$
\sigma_{R}(D) \leq 0 .
$$

Proof. See Lemma 8.10 and Theorem 8.4.

Lemma 20.5. $\tau_{R}(D) \geq 0$, for all standard clusters $R$.

Proof. This is Lemma 10.1.

Recall that $v$ has type $(p, q)$ if every standard region with a vertex at $v$ is a triangle or a quadrilateral, and if there are exactly $p$ triangular faces and $q$ quadrilateral faces that meet at $v$ (see Definition 18.6). We write $\left(p_{v}, q_{v}\right)$ for the type of $v$. Define constants $\tau_{\mathrm{LP}}(p, q) / p t$ by Table 20.1. The entries marked with an asterisk will not be needed.

Lemma 20.6. Let $S_{1}, \ldots, S_{p}$ and $R_{1}, \ldots, R_{q}$ be the tetrahedra and quad clusters around a vertex of type $(p, q)$. Consider the constants of Table 20.1. Now,

$$
\sum^{p} \tau\left(S_{i}\right)+\sum^{q} \tau\left(R_{i}\right) \geq \tau_{\mathrm{LP}}(p, q) .
$$

Proof. This is Lemma 10.5 .

Lemma 20.7. Let $v_{1}, \ldots, v_{k}$, for some $k \leq 4$, be distinct vertices of type $(5,0)$. Let $S_{1}, \ldots, S_{r}$ be quasi-regular tetrahedra around the edges $\left(0, v_{i}\right)$, for $i \leq k$. Then

$$
\sum_{i=1}^{r} \tau\left(S_{i}\right)>0.55 k p t
$$

Table 20.1. $\tau_{\mathrm{LP}}(p, q) / p t$.

\begin{tabular}{cccccccc}
\hline \multicolumn{7}{c}{$q$} \\
\cline { 2 - 8 }$p$ & 0 & 1 & 2 & 3 & 4 & 5 & 6 \\
\hline & $*$ & $*$ & 15.18 & 7.135 & 10.6497 & 22.27 \\
1 & $*$ & $*$ & 6.95 & 7.135 & 17.62 & 32.3 \\
2 & $*$ & 8.5 & 4.756 & 12.9814 & $*$ & $*$ & \\
3 & $*$ & 3.6426 & 8.334 & 20.9 & $*$ & $*$ \\
4 & 4.1396 & 3.7812 & 16.11 & $*$ & $*$ & $*$ \\
5 & 0.55 & 11.22 & $*$ & $*$ & $*$ & $*$ \\
6 & 6.339 & $*$ & $*$ & $*$ & $*$ & $*$ \\
7 & 14.76 & $*$ & $*$ & $*$ & $*$ & $*$ \\
\hline
\end{tabular}


and

$$
\sum_{i=1}^{r} \sigma\left(S_{i}\right)<r p t-0.48 k p t .
$$

Proof. This is Lemma 10.6.

Lemma 20.8. Let $D$ be a contravening decomposition star. If the type of the vertex is ( $p, q, r)$ with $r=0$, then $(p, q)$ must be one of the following:

$$
\begin{array}{r}
\{(6,0),(5,0),(4,0),(5,1),(4,1),(3,1),(2,1), \\
(3,2),(2,2),(1,2),(2,3),(1,3),(0,3),(0,4)\} .
\end{array}
$$

Proof. This is Lemma 10.10 and Corollary 12.3.

Lemma 20.9. A triangular standard region does not contain any enclosed vertices.

Proof. This fact is proved in Lemma 3.7 of [Ha6].

Lemma 20.10. A quadrilateral region does not enclose any vertices of height at most $2 t_{0}$.

Proof. This is Lemma 10.13.

Lemma 20.11. Let $F$ be a union of standard regions. Suppose that the boundary of $F$ consists of four edges. Suppose that the area of $F$ is at most $2 \pi$. Then there is at most one enclosed vertex over $F$.

Proof. This is Proposition 4.2 of [Ha6].

Lemma 20.12. Let $F$ be the union of two standard regions, a triangular region and a pentagonal region that meet at a vertex of type $(1,0,1)$ as shown in Fig. 20.2. Then

$$
\tau_{F}(D) \geq 11.16 p t .
$$

Proof. This is Lemma 14.4.

Lemma 20.13. Let $R$ be an exceptional standard region. Suppose that $R$ has $r$ different interior angles that are pairwise nonadjacent and such that each is at most 1.32 . Then

$$
\tau_{R}(D) \geq t_{n}+r(1.47) p t .
$$

Proof. This is Remark 14.2. 




Fig. 20.2. A 4-circuit.

Lemma 20.14. Every interior angle of every standard region is at least 0.8638 . Every interior angle of every standard region that is not a triangle is at least 1.153.

Proof. CALC-208809199 and CALC-853728973-1.

Definition 20.15. The central vertex of a flat quarter is defined to be the one that does not lie on the triangle formed by the origin and the diagonal.

Lemma 20.16. If the interior angle at a corner $v$ of a nontriangular standard region is at most 1.32, then there is a flat quarter over $R$ whose central vertex is $v$.

Proof. This is Lemma 11.30.

\subsection{Contravening Plane Graphs Defined}

A plane graph $G$ is attached to every contravening decomposition star as follows. From the decomposition star $D$, it is possible to determine the coordinates of the set $U(D)$ of vertices at distance at most $2 t_{0}$ from the origin.

If we draw a geodesic arc on the unit sphere at the origin with endpoints at the radial projections of $v_{1}$ and $v_{2}$ for every pair of vertices $v_{1}, v_{2} \in U(D)$ such that $\left|v_{1}\right|,\left|v_{2}\right|,\left|v_{1}-v_{2}\right| \leq 2 t_{0}$, we obtain a plane graph that breaks the unit sphere into standard regions. (The arcs do not meet except at endpoints by Lemma 4.19.)

For a given standard region, we consider the arcs forming its boundary together with the arcs that are internal to the standard region. We consider the points on the unit sphere formed by the endpoints of the arcs, together with the radial projections to the unit sphere of vertices in $U$ whose radial projection lies in the interior of the region.

Remark 20.17. The system of arcs and vertices associated with a standard region in a contravening example must be a polygon, or one of the configurations of Fig. 20.1 (see Lemma 20.1). 


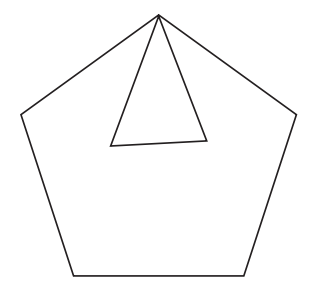

Fig. 20.3. An aggregate forming a pentagon.

Remark 20.18. Observe that one case of Fig. 20.1 is bounded by a triangle and a pentagon, and that the others are bounded by a polygon. Replacing the triangle-pentagon arrangement with the bounding pentagon and replacing the others with the bounding polygon, we obtain a partition of the sphere into simple polygons. Each of these polygons is a single standard region, except in the triangle-pentagon case (Fig. 20.3), which is a union of two standard regions (a triangle and an eight-sided region).

Remark 20.19. To simplify further, if we have an arrangement of six standard regions around a vertex formed from five triangles and one pentagon, we replace it with the bounding octagon (or hexagon). See Fig. 20.4. (It will be shown in Lemma 21.11 that there is at most one such configuration in the standard decomposition of a contravening decomposition star, so we will not worry here about how to treat the case of two overlapping configurations of this sort.)

In summary, we have a plane graph that is approximately that given by the standard regions of the decomposition star, but simplified to a bounding polygon when one of the configurations of Remarks 20.18 and 20.19 occur. We refer to the combination of standard regions into a single face of the graph as aggregation. We call it the plane graph $G=G(D)$ attached to a contravening decomposition star $D$.

Proposition 21.1 will show the vertex set $U$ is nonempty and that the graph $G(D)$ is nonempty.

When we refer to the plane graph in this manner, we mean the combinatorial plane graph as opposed to the embedded metric graph on the unit sphere formed from the system of geodesic arcs. Given a vertex $v$ in $G(D)$, there is a uniquely determined vertex $v(D)$ of $U(D)$ whose radial projection to the unit sphere determines $v$. We call $v(D)$ the corner in $U(D)$ over $v$.

By construction, the plane graphs associated with a decomposition star do not have loops or multiple joins. In fact, the edges of $G(D)$ are defined by triangles whose sides



Fig. 20.4. Degree 6 aggregates. 
vary between lengths 2 and $2 t_{0}$. The angles of such a triangle are strictly less than $\pi$. This implies that the edges of the metric graph on the unit sphere always have an arclength strictly less than $\pi$. In particular, the endpoints are never antipodal. A loop on the combinatorial graph corresponds to an edge on the metric graph that is a closed geodesic. A multiple join on the combinatorial graph corresponds on the metric graph to a pair of points joined by multiple minimal geodesics, that is, a pair of antipodal points on the sphere. By the arc-length constraints on edges in the metric graph, there are no loops or multiple joins in the combinatorial graph $G(D)$.

In Section 18.3 a plane graph satisfying a certain restrictive set of properties is said to be tame. If a plane graph $G(D)$ is associated with a contravening decomposition star $D$, we call $G(D)$ a contravening plane graph.

Theorem 20.20. Let $D$ be a contravening decomposition star. Then its plane graph $G(D)$ is tame.

This theorem is one of the main steps in the proof of the Kepler conjecture. It is advanced as one of the central claims in Section 3.3. Its proof occupies Sections 21 and 22. In Theorem 19.1 the tame graphs are classified up to isomorphism. As a corollary, we have an explicit list of graphs that contains all contravening plane graphs.

\section{Contravention is tame}

This section begins the proof of Theorem 20.20 (contravening graphs are tame). To prove Theorem 20.20, it is enough to show that each defining property of tameness is satisfied for every contravening graph. This is the substance of results in the following sections. The proof continues through to the end of Section 22. This section verifies all the properties of tameness, except for the last one (weight assignments).

\subsection{First Properties}

This section verifies Properties 1, 2, 4, and 8 of tameness. First, we prove the promised nondegeneracy result.

Proposition 21.1. The construction of Section 20.2 associates a (nonempty) plane graph with at least two faces to every decomposition star $D$ with $\sigma(D)>0$.

Proof. First we show that decomposition stars with $\sigma(D)>0$ have nonempty vertex sets $U$. (Recall that $U$ is the set of vertices of distance at most $2 t_{0}$ from the center.) The vertices of $U$ are used in Sections 4 and 5 to create all of the structural features of the decomposition star: quasi-regular tetrahedra, quarters, and so forth. If $U$ is empty, the $V$-cell is a solid containing the ball $B\left(t_{0}\right)$ of radius $t_{0}$, and $\sigma(D)$ satisfies

$$
\begin{aligned}
\sigma(D) & =\operatorname{vor}(D) \\
& =-4 \delta_{\mathrm{oct}} \operatorname{vol}(\operatorname{VC}(D))+4 \pi / 3 \\
& <-4 \delta_{\mathrm{oct}} \operatorname{vol}\left(B\left(t_{0}\right)\right)+4 \pi / 3<0 .
\end{aligned}
$$

By hypothesis, $\sigma(D)>0$. So $U$ is not empty. 
Equation (20.5) shows that the function $\sigma$ can be expressed as a sum of terms $\sigma_{R}$ indexed by the standard regions $R$. It is proved in Theorem 8.4 that $\sigma_{R} \leq 0$, unless $R$ is a triangle. Thus, a decomposition star with positive $\sigma(D)$ must have at least one triangle. Its complement contains a second standard region. Even after we form aggregates of distinct standard regions to form the simplified plane graph (Remarks 20.18 and 20.19), there certainly remain at least two faces.

Proposition 21.2. The plane graph of a contravening decomposition star satisfies Property 1 of tameness: The length of each face is at least 3 and at most 8.

Proof. By the construction of the graph, each face has at least three edges. The upper bound of eight edges is Lemma 20.1. Note that the aggregates of Remarks 20.19 and 20.18 have between five and eight edges.

Proposition 21.3. The plane graph of a contravening decomposition star satisfies Property 2 of tameness: Every 3-circuit is a face or the opposite of a face.

Proof. The simplifications of the plane graph in Remarks 20.18 and 20.19 do not produce any new 3-circuits. (See the accompanying figures.) The result is Lemma 20.9.

Proposition 21.4. Contravening graphs satisfy Property 4 of tameness: The degree of every vertex is at least 2 and at most 6.

Proof. The statement that degrees are at least 2 trivially follows because each vertex lies on at least one polygon, with two edges at that vertex.

If the type is $(p, q)$, then the impossibility of a vertex of degree 7 or more is found in Lemma 20.8. If the type is $(p, q, r)$, with $r \geq 1$, then Lemma 20.14 shows that the interior angles of the standard regions cannot sum to $2 \pi$ :

$$
6(0.8638)+1.153>2 \pi \text {. }
$$

Proposition 21.5. Contravening graphs satisfy Property 8 of tameness: There are never two vertices of type $(4,0)$ that are adjacent to each other.

Proof. This is proved in Proposition 4.2 of [Ha6].

\subsection{Computer Calculations and Their Consequences}

This section continues in the proof that all contravening plane graphs are tame. The next few sections verify Properties 6, 5, and then 3 of tameness.

In this section we rely on some inequalities that are not proved in this paper. Recall from Section 8.3 that there is an archive of hundreds of inequalities that have been proved by computer. This full archive appears in [Ha16]. The justification of these inequalities appears in the same archive. (The proofs of these inequalities were executed by computer.) 
Each inequality carries a nine digit identifying number. To invoke an inequality, we state it precisely, and give its identifying number, e.g., CALC-123456789.

To use these inequalities systematically, we combine inequalities into linear programs and solve the linear programs on computer. At first, our use of linear programs will be light, but our reliance will become progressively strong as the argument develops.

To start out, we make use of several calculations ${ }^{132}$ that give lower bounds on $\tau_{R}(D)$ when $R$ is a triangle or a quadrilateral. To obtain lower bounds through linear programming, we take a linear relaxation. Specifically, we introduce a linear variable for each function $\tau_{R}$ and a linear variable for each interior angle $\alpha_{R}$. We substitute these linear variables for the nonlinear functions $\tau_{R}(D)$ and nonlinear interior angle function into the given inequalities. Under these substitutions, the inequalities become linear. Given $p$ triangles and $q$ quadrilaterals at a vertex, we have the linear program to minimize the sum of the (linear variables associated with) $\tau_{R}(D)$ subject to the constraint that the (linear variables associated with the) angles at the vertex sum to at most $d$. Linear programming yields ${ }^{133}$ a lower bound $\tau_{\mathrm{LP}}(p, q, d)$ to this minimization problem. This gives a lower bound to the corresponding constrained sum of nonlinear functions $\tau_{R}$.

Similarly, another group of inequalities ${ }^{134}$ yields upper bounds $\sigma_{\mathrm{LP}}(p, q, d)$ on the sum of $p+q$ functions $\sigma_{R}$, with $p$ standard regions $R$ that are triangular, and another $q$ that are quadrilateral. These linear programs find their first application in the proof of the following proposition.

\subsection{Linear Programs}

To continue with the proof that contravening plane graphs are tame, we need to introduce more notation and methods.

If $F$ is a face of $G(D)$, let

$$
\sigma_{F}(D)=\sum \sigma_{R}(D)
$$

where the sum runs over the set of standard regions associated with $F$. This sum reduces to a single term unless $F$ is an aggregate in the sense of Remarks 20.19 and 20.18.

Lemma 21.6. The plane graph of a contravening decomposition star satisfies Property 6 of tameness:

$$
\sum_{F} c(\operatorname{len}(F)) \geq 8
$$

Proof. We will show that

$$
c(\operatorname{len}(F)) p t \geq \sigma_{F}(D) .
$$

\footnotetext{
132 The sequence of five inequalities starting with CALC-927432550, Lemma 20.5, and for quads CALC-310151857, CALC-655029773, CALC-73283761, CALC-15141595, CALC-574391221, CALC-396281725.

133 Although they are closely related, the function $\tau_{\mathrm{LP}}$ of three arguments introduced here is distinct from the function of two variables of the same name that is introduced in Section 20.1.

134 CALC-539256862, CALC-864218323, CALC-776305271, and for quads CALC-310151857, CALC-655029773, CALC-73283761, CALC-15141595, CALC-574391221, CALC-396281725.
} 
Assuming this, the result follows for contravening stars $D$ :

$$
\begin{aligned}
\sum_{F} c(\operatorname{len}(F)) p t & \geq \sum_{F} \sigma_{F}(D) \\
& =\sigma(D) \geq 8 p t .
\end{aligned}
$$

We consider three cases for inequality (21.1). In the first case assume that the face $F$ corresponds to exactly one standard region in the decomposition star. In this case inequality (21.1) follows directly from the bounds of Lemma 20.2:

$$
\sigma_{F}(D) \leq s_{n} \leq c(n) p t .
$$

In the second case assume the context of a pentagon $F$ formed in Remark 20.18. Then, again by Lemma 20.2, we have

$$
\sigma_{F}(D) \leq s_{3}+s_{8} \leq(c(3)+c(8)) p t \leq c(5) p t .
$$

(Just examine the constants $c(k)$.)

In the third case we consider the situation of Remark 20.19. The six standard regions give

$$
\sigma_{F}(D) \leq s_{5}+\sigma_{\mathrm{LP}}(5,0,2 \pi-1.153)<c(8) p t .
$$

The constant 1.153 comes from Lemma 20.14 .

Proposition 21.7. Let $F$ be a face of a contravening plane graph $G(D)$. Then

$$
\tau_{F}(D) \geq d(\operatorname{len}(F)) p t .
$$

Proof. Similar.

Lemma 21.8. If $v$ is a vertex of an exceptional standard region, and if there are six standard regions meeting at $v$, then the exceptional region is a pentagonal region and the other five standard regions are triangular.

Proof. There are several cases according to the number $k$ of triangular regions at the vertex.

$(k \leq 2)$ If there are at least four nontriangular regions at the vertex, then the sum of interior angles around the vertex is at least $4(1.153)+2(0.8638)>2 \pi$, which is impossible. (See Lemma 20.14.)

$(k=3)$ If there are three nontriangular regions at the vertex, then $\tau(D)$ is at least $2 t_{4}+t_{5}+\tau_{\mathrm{LP}}(3,0,2 \pi-3(1.153))>(4 \pi \zeta-8) p t$.

$(k=4)$ If there are two exceptional regions at the vertex, then $\tau(D)$ is at least $2 t_{5}+\tau_{\mathrm{LP}}(4,0,2 \pi-2(1.153))>(4 \pi \zeta-8) p t$.

If there are two nontriangular regions at the vertex, then $\tau(D)$ is at least $t_{5}+$ $\tau_{\mathrm{LP}}(4,1,2 \pi-1.153)>(4 \pi \zeta-8) p t$.

$(k=5)$ We are left with the case of five triangular regions and one exceptional region. 


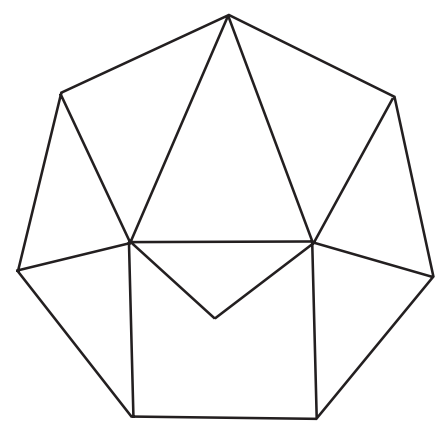

Fig. 21.1. Nonadjacent vertices of degree 6 on a pentagon.

When there is an exceptional standard region at a vertex of degree 6 , we claim that the exceptional region must be a pentagon. If the region is a heptagon or more, then $\tau(D)$ is at least $t_{7}+\tau_{\mathrm{LP}}(5,0,2 \pi-1.153)>(4 \pi \zeta-8) p t$.

If the standard region is a hexagon, then $\tau(D)$ is at least $t_{6}+\tau_{\mathrm{LP}}(5,0,2 \pi-1.153)>t_{9}$. Also, $s_{6}+\sigma_{\mathrm{LP}}(5,0,2 \pi-1.153)<s_{9}$. The aggregate of the six standard regions is ninesided. Lemma 20.3 gives the bound of $8 p t$.

Lemma 21.9. Consider the standard regions of a contravening star D.

1. If a vertex of a pentagonal standard region has degree 6 , then the aggregate $F$ of the six faces satisfies

$$
\begin{gathered}
\sigma_{F}(D)<s_{8}, \\
\tau_{F}(D)>t_{8} .
\end{gathered}
$$

2. An exceptional standard region has at most two vertices of degree 6. If there are two, then they are nonadjacent vertices on a pentagon, as shown in Fig. 21.1.

Proof. We begin with the first part of the lemma. The sum $\tau_{F}(D)$ over these six standard regions is at least

$$
t_{5}+\tau_{\mathrm{LP}}(5,0,2 \pi-1.153)>t_{8}
$$

Similarly,

$$
s_{5}+\sigma_{\mathrm{LP}}(5,0,2 \pi-1.153)<s_{8} .
$$

We note that there can be at most one exceptional region with a vertex of degree 6 . Indeed, if there are two, then they must both be vertices of the same pentagon:

$$
t_{8}+t_{5}>(4 \pi \zeta-8) p t
$$

Such a second vertex on the octagonal aggregate leads to one of the following constants greater than $(4 \pi \zeta-8) p t$. These same constants show that such a second vertex on a 
hexagonal aggregate must share two triangular faces with the first vertex of degree 6 .

$$
\begin{aligned}
& t_{8}+\tau_{\mathrm{LP}}(4,0,2 \pi-1.32-0.8638), \quad \text { or } \\
& t_{8}+1.47 p t+\tau_{\mathrm{LP}}(4,0,2 \pi-1.153-0.8638), \quad \text { or } \\
& t_{8}+\tau_{\mathrm{LP}}(5,0,2 \pi-1.153) .
\end{aligned}
$$

(The relevant constants are found at Lemmas 20.13 and 20.14.)

\subsection{A Noncontravening 4-Circuit}

This subsection rules out the existence of a particular 4-circuit on a contravening plane graph. The interior of the circuit consists of two faces: a triangle and a pentagon. The circuit and its enclosed vertex are show in Fig. 20.2 with vertices marked $p_{1}, \ldots, p_{5}$. The vertex $p_{1}$ is the enclosed vertex, the triangle is $\left(p_{1}, p_{2}, p_{5}\right)$, and the pentagon is $\left(p_{1}, \ldots, p_{5}\right)$. Let $v_{1}, \ldots, v_{4}, v_{5}$ be the corresponding vertices of $U(D)$.

The diagonals $\left\{v_{5}, v_{3}\right\}$ and $\left\{v_{2}, v_{4}\right\}$ have length at least $2 \sqrt{2}$ by Lemma 4.19 . If an interior angle of the quadrilateral is less than 1.32 , then, by Lemma $20.16,\left|v_{1}-v_{3}\right| \leq \sqrt{8}$. Thus, we assume in the following lemma that all interior angles of the quadrilateral aggregate are at least 1.32 .

Lemma 21.10. A decomposition star that contains this configuration does not contravene.

Proof. Let $P$ denote the quadrilateral aggregate of these two standard regions. By Lemma 20.12 we have $\tau_{P}(D) \geq 11.16 p t$. There are no other exceptional faces, because $11.16 p t+t_{5}>(4 \pi \zeta-8) p t$. Every vertex not on $P$ has type $(5,0)$, by Lemma 20.6. In particular, there are no quadrilateral regions. The interior angles of $P$ are at least 1.32. There are at most four triangles at every vertex of $P$, because

$$
11.16 p t+\tau_{\mathrm{LP}}(5,0,2 \pi-1.32)>(4 \pi \zeta-8) p t .
$$

There are at least three triangles at every vertex of $P$, otherwise we contradict Lemmas 20.9 or 20.11 .

The only triangulation with these properties is obtained by removing one edge from the icosahedron (Exercise). This implies that there are two opposite corners of $P$ each having four quasi-regular tetrahedra. Since the diagonals of $P$ have lengths greater than $2 \sqrt{2}$, the results of CALC-325738864 show that the union $F$ of these eight quasi-regular tetrahedra satisfies

$$
\tau_{F}(D) \geq 2(1.5) p t .
$$

There are two additional vertices of type $(5,0)$ whose tetrahedra are distinct from these eight quasi-regular tetrahedra. They give an additional 2(0.55) $p t$. Now $(11.16+2(1.5)+$ $2(0.55)) p t>(4 \pi \zeta-8) p t$ by Lemma 20.7. The result follows.

Lemma 21.11. A contravening plane graph satisfies Property 5 of tameness: If a vertex is contained in an exceptional face, then the degree of the vertex is at most five. 
Proof. An exceptional standard region with a vertex of degree 6 must be pentagonal by Lemma 21.9. If that pentagonal region has two or more such vertices, then by the same lemma it must be the arrangement shown in Fig. 21.1. This arrangement does not appear on a contravening graph by Lemma 21.10 .

Remark 21.12. We have now fully justified the claim made in Remark 20.19: there is at most one vertex on six standard regions, and it is part of an aggregate in such a way that it does not appear as the vertex of $G(D)$.

\subsection{Possible 4-Circuits}

Every 4-circuit divides a plane graph into two aggregates of faces that we may call the interior and exterior. We call vertices of the faces in the aggregate that do not lie on the 4-cycle enclosed vertices. Thus, every vertex lies in the 4-cycle, is enclosed over the interior, or is enclosed over the exterior.

Lemma 20.11 asserts that either the interior or the exterior has at most one enclosed vertex. When choosing which aggregate is to be called the interior, we may make our choice so that the interior has area at most $2 \pi$, and hence contains at most one vertex. With this choice, we have the following proposition.

Proposition 21.13. Let $D$ be a contravening plane graph. A 4-circuit surrounds one of the aggregates of faces shown in Property 3 of tameness.

Proof. If there are no enclosed vertices, then the only possibilities are for it to be a single quadrilateral face or a pair of adjacent triangles.

Assume there is one enclosed vertex $v$. If $v$ is connected to three or four vertices of the quadrilateral, then that possibility is listed as part of the conclusion.

If $v$ is connected to two opposite vertices in the 4-cycle, then the vertex $v$ has type $(0,2)$ and the bounds of Lemma 20.6 show that the graph cannot be contravening.

If $v$ is connected to two adjacent vertices in the 4-cycle, then we appeal to Lemma 21.10 to conclude that the graph does not contravene.

If $v$ is connected to at most one vertex, then we appeal to Lemma 20.10. This completes the proof.

\section{Weight Assignments}

The purpose of this section is to prove the existence of a good admissible weight assignment for contravening plane graphs. This will complete the proof that all contravening graphs are tame.

Theorem 22.1. Every contravening plane graph has an admissible weight assignment of total weight less than $\operatorname{tgt}=14.8$. 
Given a contravening decomposition star $D$, we define a weight assignment $w$ by

$$
F \mapsto w(F)=\tau_{F}(D) / p t .
$$

Since $D$ contravenes,

$$
\begin{aligned}
\sum_{F} w(F) & =\sum_{F} \tau_{F}(D) / p t \\
& =\tau(D) / p t \\
& \leq(4 \pi \zeta-8) p t / p t \\
& <\operatorname{tgt}=14.8 .
\end{aligned}
$$

The challenge of the theorem will be to prove that $w$, when defined by this formula, is admissible.

\subsection{Admissibility}

The next three lemmas establish that this definition of $w(F)$ for contravening plane graphs satisfies the first three defining properties of an admissible weight assignment.

Lemma 22.2. Let $F$ be a face of length $n$ in a contravening plane graph. Define $w(F)$ as above. Then $w(F) \geq d(n)$.

Proof. This is Proposition 21.7.

Lemma 22.3. Let $v$ be a vertex of type $(p, q)$ in a contravening plane graph. Define $w(F)$ as above. Then

$$
\sum_{v \in F} w(F) \geq b(p, q)
$$

Proof. This is Lemma 20.6.

Lemma 22.4. Let $V$ be any set of vertices of type $(5,0)$ in a contravening plane graph. Define $w(F)$ as above. If the cardinality of $V$ is $k \leq 4$, then

$$
\sum_{V \cap F \neq \emptyset} w(F) \geq 0.55 k
$$

Proof. This is Lemma 20.7.

The following proposition establishes the final property that $w(F)$ must satisfy to make it admissible. Separated sets are defined in Section 18.2. 
Proposition 22.5. Let $V$ be any separated set of vertices in a contravening plane graph. Define $w(F)$ as above. Then

$$
\sum_{V \cap F \neq \emptyset}(w(F)-d(\operatorname{len}(F))) \geq \sum_{v \in V} a(\operatorname{tri}(v)),
$$

where tri $(v)$ denotes the number of triangles containing the vertex $v$.

The proof occupies the rest of this section. Since the degree of each vertex is 5 , and there is at least one face that is not a triangle at the vertex, the only constants $\operatorname{tri}(v)$ that arise are

$$
\operatorname{tri}(v) \in\{0, \ldots, 4\} .
$$

We will prove that in a contravening plane graph Conditions 1 and 4 of a separated set are incompatible with the condition tri $(v) \leq 2$, for some $v \in V$. This will allows us to assume that

$$
\operatorname{tri}(v) \in\{3,4\},
$$

for all $v \in V$. These cases are treated in Section 22.3.

First we prove the inequality when there are no aggregates involved. Afterwards, we show that the conclusions can be extended to aggregate faces as well.

\subsection{Proof that $\operatorname{tri}(v)>2$}

In this subsection $D$ is a contravening decomposition star with associated graph $G(D)$. Let $V$ be a separated set of vertices in $G(D)$. Let $v$ be a vertex in $V$ such that none of its faces is an aggregate in the sense of Remarks 20.18 and 20.19.

Lemma 22.6. Under these conditions, for every $v \in V, \operatorname{tri}(v)>1$.

Proof. If there are $p$ triangles, $q$ quadrilaterals, and $r$ other faces, then

$$
\begin{aligned}
\tau(D) & \geq \sum_{v \in R} \tau_{R}(D) \\
& \geq r t_{5}+\tau_{\mathrm{LP}}(p, q, 2 \pi-r(1.153)) .
\end{aligned}
$$

If there is a vertex $w$ that is not on any of the faces containing $v$, then the sum of $\tau_{F}(D)$ over the faces containing $w$ yields an additional $0.55 p t$ by Lemma 20.7. We calculate these constants for each $(p, q, r)$ and find that the bound is always greater than $(4 \pi \zeta-8) p t$. This implies that $D$ cannot be contravening.

\begin{tabular}{lll}
\hline \multicolumn{1}{c}{$(p, q, r)$} & Lower bound & Justification \\
\hline$(0,5,0)$ & $22.27 p t$ & Lemma 20.6 \\
$(0, q, r \geq 1)$ & $t_{5}+4 t_{4} \approx 14.41 p t$ & \\
$(1,4,0)$ & $17.62 p t$ & Lemma 20.6 \\
$(1,3,1)$ & $t_{5}+12.58 p t$ & $\left(\tau_{\mathrm{LP}}\right)$ \\
$(1,2,2)$ & $2 t_{5}+7.53 p t$ & $\left(\tau_{\mathrm{LP}}\right)$ \\
$(1, q, r \geq 3)$ & $3 t_{5}+t_{4}$ & \\
\hline
\end{tabular}


Lemma 22.7. Under these same conditions, for every $v \in V, \operatorname{tri}(v)>2$.

Proof. Assume that $\operatorname{tri}(v)=2$. We will show that this implies that $D$ does not contravene. Let $e$ be the number of exceptional faces at $v$. We have $e+\operatorname{tri}(v) \leq 5$.

The constants $0.55 \mathrm{pt}$ and $0.48 \mathrm{pt}$ used throughout the proof come from Lemma 20.7. The constants $t_{n}$ comes from Lemma 20.2.

$(e=3)$ First, assume that there are three exceptional faces around vertex $v$. They must all be pentagons $\left(2 t_{5}+t_{6}>(4 \pi \zeta-8) p t\right)$. The aggregate of the five faces is an $m$-gon (some $m \leq 11$ ). If there is a vertex not on this aggregate, use $3 t_{5}+0.55 p t>(4 \pi \zeta-8) p t$. So there are at most nine triangles away from the aggregate, and

$$
\sigma(D) \leq 9 p t+\left(3 s_{5}+2 p t\right)<8 p t .
$$

The argument is the same if there is a quad, a pentagon, or a hexagon $\left(t_{4}+t_{6}=\right.$ $\left.2 t_{5}, s_{4}+s_{6}=2 s_{5}\right)$.

$(e=2)$ Assume next that there are two pentagons and a quadrilateral around the vertex. The aggregate of the two pentagons, quadrilateral, and two triangles is an $\mathrm{m}$-gon (some $m \leq 10$ ). There must be a vertex not on the aggregate of five faces, for otherwise we have

$$
\sigma(D) \leq 8 p t+\left(2 s_{5}+2 p t\right)<8 p t .
$$

The interior angle of one of the pentagons is at most 1.32. For otherwise, $\tau_{\mathrm{LP}}(2,1,2 \pi-2(1.32))+2 t_{5}+0.55 p t>(4 \pi \zeta-8) p t$.

Lemma 20.13 shows that any pentagon $R$ with an interior angle less than 1.32 yields $\tau_{R}(D) \geq t_{5}+(1.47 p t)$. If both pentagons have an interior angle $<1.32$ the lemma follows easily from this calculation: $2\left(t_{5}+1.47 p t\right) p t+\tau_{\mathrm{LP}}(2,1,2 \pi-2(1.153))+$ $0.55 p t>(4 \pi \zeta-8) p t$. If there is one pentagon with an angle $>1.32$, we then have $t_{5}+(1.47 p t)+\tau_{\mathrm{LP}}(2,1,2 \pi-1.153-1.32)+t_{5}+0.55 p t>(4 \pi \zeta-8) p t$.

$(e=1)$ Assume finally that there is one exceptional face at the vertex. If it is a hexagon (or more), we are done: $t_{6}+\tau_{\mathrm{LP}}(2,2,2 \pi-1.153)>(4 \pi \zeta-8) p t$. Assume it is a pentagon. The aggregate of the five faces at the vertex is bounded by an $m$-circuit (some $m \leq 9$ ). If there are no more than nine quasi-regular tetrahedra outside the aggregate, then $\sigma(D)$ is at most $(9-2(0.48)) p t+s_{5}+\sigma_{\mathrm{LP}}(2,2,2 \pi-1.153)<8 p t$ (Lemma 20.7). So we may assume that there are at least three vertices not on the aggregate.

If the interior angle of the pentagon is greater than 1.32 , then

$$
\tau_{\mathrm{LP}}(2,2,2 \pi-1.32)+3(0.55) p t+t_{5}>(4 \pi \zeta-8) p t ;
$$

if it is less than 1.32, by Lemma 20.13

$$
\tau_{\mathrm{LP}}(2,2,2 \pi-1.153)+3(0.55) p t+1.47 p t+t_{5}>(4 \pi \zeta-8) p t .
$$

Lemma 22.8. The bound $\operatorname{tri}(v)>2$ holds if $v$ is a vertex of an aggregate face.

Proof. The exceptional region enters into the preceding two proofs in a purely formal way. Pentagons enter through the bounds

$$
t_{5}, s_{5}, 1.47 p t
$$


and angles 1.153, 1.32. Hexagons enter through the bounds

$$
t_{6}, s_{6}
$$

and so forth. These bounds hold for the aggregate faces. Hence the proofs hold for aggregates as well.

\subsection{Bounds when $\operatorname{tri}(v) \in\{3,4\}$}

In this subsection $D$ is a contravening decomposition star with associated graph $G(D)$. Let $V$ be a separated set of vertices. For every vertex $v$ in $V$, we assume that none of its faces is an aggregate in the sense of Remarks 20.18 and 20.19. We assume that there are three or four triangles containing $v$, for every $v \in V$.

To prove Inequality (4) in the definition of admissible weight assignments, we rely on the following reductions. Define an equivalence relation on exceptional faces by $F \sim F^{\prime}$ if there is a sequence $F_{0}=F, \ldots, F_{r}=F^{\prime}$ of exceptional faces such that consecutive faces share a vertex of type $(3,0,2)$. (That is, $\operatorname{tri}(v)=3$.) Let $\mathcal{F}$ be an equivalence class of faces.

Lemma 22.9. Let $V$ be a separated set of vertices. For every equivalence class of exceptional faces $\mathcal{F}$, let $V(\mathcal{F})$ be the subset of $V$ whose vertices lie in the union of faces of $\mathcal{F}$. Suppose that for every equivalence class $\mathcal{F}$, Inequality (4) (in the definition of admissible weight assignments) holds for $V(\mathcal{F})$. Then the inequality holds for $V$.

Proof. By construction, each vertex in $V$ lies in some $F$, for an exceptional face. Moreover, the separating property of $V$ ensures that the triangles and quadrilaterals in the inequality are associated with a well-defined $\mathcal{F}$. Thus, the inequality for $V$ is a sum of the inequalities for each $V(\mathcal{F})$.

Lemma 22.10. Let $v$ be a vertex in a separated set $V$ at which there are p triangles, $q$ quadrilaterals, and $r$ other faces. Suppose that for some $p^{\prime} \leq p$ and $q^{\prime} \leq q$, we have

$$
\tau_{\mathrm{LP}}\left(p^{\prime}, q^{\prime}, \alpha\right)>\left(p^{\prime} d(3)+q^{\prime} d(4)+a(p)\right) p t
$$

for some upper bound $\alpha$ on the angle occupied by $p^{\prime}$ triangles and $q^{\prime}$ quadrilaterals at $v$. Suppose further that Inequality 4 (in the definition of admissible weight assignments) holds for the separated set $V^{\prime}=V \backslash\{v\}$. Then the inequality holds for $V$.

Proof. Let $F_{1}, \ldots, F_{m}, m=p^{\prime}+q^{\prime}$, be faces corresponding to the triangles and quadrilaterals in the lemma. The hypotheses of the lemma imply that

$$
\sum_{1}^{m}\left(w F_{i}(D)-d\left(\operatorname{len}\left(F_{i}\right)\right)\right)>a(p)
$$

Clearly, the inequality for $V$ is the sum of this inequality, the inequality for $V^{\prime}$, and $d(n) \geq 0$. 
Recall that the central vertex of a flat quarter is defined to be the one that does not lie on the triangle formed by the origin and the diagonal.

Lemma 22.11. Let $R$ be an exceptional standard region. Let $V$ be a set of vertices of $R$. If $v \in V$, let $p_{v}$ be the number of triangular regions at $v$ and let $q_{v}$ be the number of quadrilateral regions at $v$. Assume that $V$ has the following properties:

1. The set $V$ is separated.

2. If $v \in V$, then there are five standard regions at $v$.

3. If $v \in V$, then the corner over $v$ is a central vertex of a flat quarter in the cone over $R$.

4. If $v \in V$, then $p_{v} \geq 3$. That is, at least three of the five standard regions at $v$ are triangular.

5. If $R^{\prime} \neq R$ is an exceptional region at $v$, and if $R$ has an interior angle at least 1.32 at $v$, then $R^{\prime}$ also has an interior angle at least 1.32 at $v$.

Let $F$ be the union of $\{R\}$ with the set of triangular and quadrilateral regions that have a vertex at some $v \in V$. Then

$$
\tau_{F}(D)>\sum_{v \in V}\left(p_{v} d(3)+q_{v} d(4)+a\left(p_{v}\right)\right) p t .
$$

Proof. If $\left(p_{v}, q_{v}\right)=(3,1)$ and the internal angle of $R$ at $v$ is at least 1.32 , then we use

$$
\tau_{\mathrm{LP}}(3,1,2 \pi-1.32)>1.4 p t+t_{4} .
$$

In this case the inequality of the lemma is a consequence of this inequality and the inequality for $V \backslash\{v\}$. Thus, we may assume without loss of generality that if $\left(p_{v}, q_{v}\right)=$ $(3,1)$, then the internal angle of $R$ at $v$ is at most 1.32. The conclusion now follows from Lemma 14.6.

Lemma 22.12. Property 4 of admissibility holds. That is, let $V$ be any separated set of vertices. Then

$$
\sum_{F: V \cap F \neq \emptyset}(w(F)-d(\operatorname{len}(F))) \geq \sum_{v \in V} a(\operatorname{tri}(v))
$$

Proof. Let $V$ be a separated set of vertices. The results of Section 22.2 reduce the lemma to the case where $\operatorname{tri}(v) \in\{3,4\}$ for every vertex $v \in V$.

We say that there is a flat quarter centered at $v$, if the corner $v^{\prime}$ over $v$ is the central vertex of a flat quarter and that flat quarter lies in the cone over an exceptional region.

One case is easy to deal with. Assume that there are three triangles, a quadrilateral, and an exceptional face at the vertex. Assume the interior angle on the exceptional region is at least 1.32 ; then

$$
\tau_{\mathrm{LP}}(3,1,2 \pi-1.32)>1.4 p t+t_{4} .
$$

This gives the bound in the sense of Lemma 22.10 at such a vertex. For the rest of the proof, assume that the interior angle on the exceptional region is less than 1.32 at vertices 
of type $(p, q, r)=(3,1,1)$. This implies in particular by Lemma 20.16 that there is a flat quarter centered at each vertex of this type.

Let $v$ be a vertex with no flat quarter centered at $v$. By Lemma 20.16, the interior angles of the exceptional regions at $v$ are at least 1.32 . It follows ${ }^{135}$ that

$$
\tau_{\mathrm{LP}}\left(p_{v}, q_{v}, \alpha\right)>\left(p_{v} d(3)+q_{v} d(4)+a\left(p_{v}\right)\right) p t
$$

Thus, by Lemma 22.10, we reduce to the case where for each $v \in V$, there is a flat quarter centered at $v$. Assume that $V$ has this property.

Pick a function $f$ from the set $V$ to the set of exceptional standard regions as follows. If there is only one exceptional region at $v$, then let $f(v)$ be that exceptional region. If there are two exceptional regions at $v$, then let $f(v)$ be one of these two exceptional regions. Pick it to be an exceptional region with an interior angle at most 1.32 if one of the two exceptional regions has this property. Pick it to have a flat quarter centered at $v$. Note that by Lemma 20.16, if the exceptional region has an interior angle at most 1.32, then $f(v)$ will have a flat quarter centered at $v$.

For each exceptional region $R$, let

$$
V_{R}=\{v \in V: f(v)=R\}
$$

By Lemma 22.11, Property 4 of admissibility is satisfied for each $V_{R}$. Since this property is additive in $V_{R}$ and since $V$ is the disjoint union of the sets $V_{R}$, the proof is complete.

\subsection{Weight Assignments for Aggregates}

Lemma 22.13. Consider a separated set of vertices $V$ on an aggregated face $F$ as in Remark 20.18. Then inequality 4 holds (in the definition of admissible weight assignments):

$$
\sum_{V \cap F \neq \emptyset}(w(F)-d(\operatorname{len}(F))) \geq \sum_{v \in V} a(\operatorname{tri}(v))
$$

Proof. We may assume that $\operatorname{tri}(v) \in\{3,4\}$.

First consider the aggregate of Remark 20.18 of a triangle and an eight-sided region, with pentagonal hull $F$. There is no other exceptional region in a contravening decomposition star with this aggregate:

$$
t_{8}+t_{5}>(4 \pi \zeta-8) p t
$$

A separated set of vertices $V$ on $F$ has cardinality at most 2. This gives the desired bound

$$
t_{8}>t_{5}+2(1.5) p t
$$

135 CALC-551665569, CALC-824762926, and CALC-325738864. 
Next, consider the aggregate of a hexagonal hull with an enclosed vertex. Again, there is no other exceptional face. If there are at most $k \leq 2$ vertices in a separated set, then the result follows from

$$
t_{8}>t_{6}+k(1.5) p t .
$$

There are at most three vertices in $V$ on a hexagon, by the nonadjacency conditions defining $V$. A vertex $v$ can be removed from $V$ if it is not the central vertex of a flat quarter (Lemma 22.10 and inequalities (22.1) and (22.2)). If there is an enclosed vertex $w$, it is impossible for there to be three nonadjacent vertices, each the central vertex of a flat quarter:

$$
\mathcal{E}\left(2,2,2, \sqrt{8}, \sqrt{8}, \sqrt{8}, 2 t_{0}, 2 t_{0}, 2\right)>2 t_{0} .
$$

( $\mathcal{E}$ is as defined in Definition 4.14.)

Finally consider the aggregate of a pentagonal hull with an enclosed vertex. There are at most $k \leq 2$ vertices in a separated set in $F$. There is no other exceptional region:

$$
t_{7}+t_{5}>(4 \pi \zeta-8) p t .
$$

The result follows from

$$
t_{7}>t_{5}+2(1.5) p t .
$$

Lemma 22.14. Consider a separated set of vertices $V$ on an aggregate face of a contravening plane graph as in Remark 20.19. Inequality 4 holds in the definition of admissible weight assignments.

Proof. There is at most one exceptional face in the plane graph:

$$
t_{8}+t_{5}>(4 \pi \zeta-8) p t
$$

Assume first that an aggregate face is an octagon (Fig. 20.4). At each of the vertices of the face that lies on a triangular standard region in the aggregate, we can remove the vertex from $V$ using Lemma 22.10 and the estimate

$$
\tau_{\mathrm{LP}}(4,0,2 \pi-2(0.8638))>1.5 p t .
$$

This leaves at most one vertex in $V$, and it lies on a vertex of $F$ which is "not aggregated," so that there are five standard regions of the associated decomposition star at that vertex, and one of those regions is pentagonal. The value $a(4)=1.5 \mathrm{pt}$ can be estimated at this vertex in the same way it is done for a nonaggregated case in Section 22.3.

Now consider the case of an aggregate face that is a hexagon (Fig. 20.4). The argument is the same: we reduce to $V$ containing a single vertex, and argue that this vertex can be treated as in Section 22.3. (Alternatively, use the fact that the pentagon-triangle combination in this aggregate has been eliminated by Lemma 21.10.)

The proof that contravening plane graphs are tame is complete. 


\section{Linear Program Estimates}

We have completed a major portion of the proof of the Kepler conjecture by proving that every contravening plane graph is tame.

The final portion of the proof of the Kepler conjecture consists in showing that tame graphs are not contravening, except for the isomorphism class of graphs isomorphic to $G_{\text {fcc }}$ and $G_{\text {hcp }}$ associated with the face-centered cubic and hexagonal close packings.

This part of the proof treats all contravening tame graphs except for the three cases $G_{\text {fcc }}, G_{\text {pent }}$, and $G_{\mathrm{hcp}}$. The two cases $G_{\mathrm{fcc}}$ and $G_{\mathrm{hcp}}$ are treated in Theorem 8.1, and the case $G_{\text {pent }}$ is treated in Paper V.

The primary tool that will be used is linear programming. The linear programs are obtained as relaxations of the original nonlinear optimization problem of maximizing $\sigma(D)$ over all decomposition stars whose associated graph is a given tame graph $G$. The upper bounds obtained through relaxation are upper bounds to the nonlinear problem.

To eliminate a tame graph, we must show that it is not contravening. By definition, this means we must show that $\sigma(D)<8 p t$. When a single linear program does not yield an upper bound under $8 \mathrm{pt}$, we branch into a sequence of linear programs that collectively imply the upper bound of $8 \mathrm{pt}$. This will call for a sequence of increasingly complex linear programs.

For each of the tame plane graphs produced in Theorem 19.1, we define a linear programming problem whose solution dominates the value of $\sigma(D)$ on the set of decomposition stars associated with the plane graph. A description of the linear programs is presented in this section.

Theorem 23.1. If the plane graph of a contravening decomposition star is isomorphic to one in the list [Ha16], then it is isomorphic to one of the following three plane graphs: the plane graph of the pentahedral prism, that of the hexagonal-close packing, or that of the face-centered cubic packing.

This theorem is one of the central claims described in Section 3.3 that lead to the proof of the Kepler conjecture.

\subsection{Relaxation}

(NLP) Let $f: P \rightarrow \mathbb{R}$ be a function on a nonempty set $P$. Consider the nonlinear maximization problem

$$
\max _{p \in P} f(p) .
$$

(LP) Consider a linear programming problem

$\max c \cdot x$

such that $A x \leq b$, where $A$ is a matrix, $b$ and $c$ are vectors of real constants, and $x$ is a vector of variables $x=\left(x_{1}, \ldots, x_{n}\right)$. We write the linear programming problem as

$$
\max (c \cdot x: A x \leq b) .
$$


An interpretation $I$ of a linear programming problem (LP) is a nonempty set $|I|$, together with an assignment $x_{i} \mapsto x_{i}^{I}$ of functions $x_{i}^{I}:|I| \rightarrow \mathbb{R}$ to variables $x_{i}$. We say the constraints $A x \leq b$ of the linear program are satisfied under the interpretation $I$ if for all $p \in|I|$,

$$
A x^{I}(p) \leq b .
$$

The interpretation $I$ is said to be a relaxation of the nonlinear program (NLP) if the following three conditions hold:

1. $P=|I|$.

2. The constraints are satisfied under the interpretation.

3. $f(p) \leq c \cdot x^{I}(p)$, for all $p \in|I|$.

Lemma 23.2. Let (LP) be a linear program with relaxation I to (NLP). Then (LP) has a feasible solution. Moreover, if (LP) is bounded above by a constant $M$, then $M$ is an upper bound on the function $f:|I| \rightarrow \mathbb{R}$.

Proof. A feasible solution is $x_{i}=x_{i}^{I}(p)$, for any $p \in|I|$. The rest is clear.

Remark 23.3. In general, it is to be expected that the interpretations $A x^{I} \leq b$ will be nonlinear inequalities on the domain $P$. In our situation, satisfaction of the constraints will be proved by interval arithmetic. Thus, the construction of an upper bound to (NLP) breaks into two tasks: to solve the linear programs and to prove the nonlinear inequalities required to satisfy the constraints.

There are many nonlinear inequalities entering into our interpretation. These have been proved by interval arithmetic on computer and are listed at [Ha16].

Remark 23.4. There is a second method of establishing the satisfaction of inequalities under an interpretation. Suppose we wish to show that the inequality $e \cdot x \leq b^{\prime}$ is satisfied under the interpretation $I$. Suppose that we have already established that a system of inequalities $A x \leq b$ is satisfied under the interpretation $I$. We solve the linear programming problem $\max (e \cdot x: A x \leq b)$. If this maximum is at most $b^{\prime}$, then the inequality $e \cdot x \leq b^{\prime}$ is satisfied under the interpretation $I$. We refer to $e \cdot x \leq b^{\prime}$ as an LP-derived inequality (with respect to the system $A x \leq b$ ).

\subsection{The Linear Programs}

Let $G$ be a tame plane graph. Let $\operatorname{DS}(G)$ be the space of all decomposition stars whose associated plane graph is isomorphic to $G$.

Theorem 23.5. For every tame plane graph $G$ other than $G_{\mathrm{fcc}}, G_{\mathrm{hcp}}$, and $G_{\mathrm{pent}}$, there exists a finite sequence of linear programs with the following properties:

1. Every linear program has an admissible solution and its solution is strictly less than 8 pt. 
2. For every linear program in this sequence, there is an interpretation I of the linear program that is a relaxation of the nonlinear optimization problem

$$
\sigma:|I| \rightarrow \mathbb{R}
$$

where $|I|$ is a subset of $\operatorname{DS}(G)$.

3. The union of the subsets $|I|$, as we run over the sequence of linear programs, is $\operatorname{DS}(G)$.

The proof is constructive. For every tame plane graph $G$ a sequence of linear programs is generated by computer and solved. The optimal solutions are all bounded above by $8 p t$. It will be clear from construction of the sequence that the union of the sets $|I|$ exhausts $\operatorname{DS}(G)$. We estimate that nearly $10^{5}$ linear programs are involved in the construction. The rest of this paper outlines the construction of some of these linear programs.

Remark 23.6. Section 3.1.1 of [Ha14] shows how the linear programs that arise in connection with the Kepler conjecture can be formulated in such a way that they always have a feasible solution and so that the optimal solution is bounded. We assume that all our linear programs have been constructed in this way.

Corollary 23.7. If a tame graph $G$ is not isomorphic to $G_{\mathrm{fcc}}, G_{\mathrm{hcp}}$, or $G_{\mathrm{pent}}$, then it is not contravening.

Proof. This follows immediately from Theorem 23.5 and Lemma 23.2.

\subsection{Basic Linear Programs}

Let $G$ be a tame plane graph. Specifically, $G$ is one of the several thousands of graphs that appear in the explicit classification [Ha16].

To describe the basic linear program, we need the following indexing sets. Let VERTEX be the set of all vertices in $G$. Let FACE be the set of all faces in $G$. (Recall that by construction each face $F$ of the graph carries an orientation.) Let ANGLE be the set of all angles in $G$, defined as the set of pairs $(v, F)$, where the vertex $v$ lies in the face $F$. Let DIRECTED be the set of directed edges. It consists of all ordered pairs $(v, s(v, F))$, where $s(v, F)$ denotes the successor of the vertex $v$ in the oriented face $F$. Let TRIANGLES be the subset of FACE consisting of those faces of length 3. Let UNDIRECTED be the set of undirected edges. It consists of all unordered pairs $\{v, s(v, F)\}$, for $v \in F$.

We introduce variables indexed by these sets. Following AMPL notation, we write for instance $y\{\operatorname{VERTEX}\}$ to declare a collection of variables $y[v]$ indexed by vertices $v$ in VERTEX. With this in mind, we declare the variables

$$
\begin{array}{lll}
\alpha\{\text { ANGLE }\}, & y\{\text { VERTEX }\}, & e\{\text { UNDIRECTED }\}, \\
\sigma\{\text { FACE }\}, & \tau\{\text { FACE }\}, & \text { sol }\{\text { FACE }\} .
\end{array}
$$

We obtain an interpretation $I$ on the compact space $\operatorname{DS}(G)$. First, we define an interpretation at the level of indexing sets. A decomposition star determines the set 
$U(D)$ of vertices of height at most $2 t_{0}$ from the origin of $D$. Each decomposition star $D \in \operatorname{DS}(G)$ determines a (metric) graph with geodesic edges on the surface of the unit sphere, which is isomorphic to $G$ as a (combinatorial) plane graph. There is a map from the vertices of $G$ to $U(D)$ given by $v \mapsto v^{I}$, if the radial projection of $v^{I}$ to the unit sphere at the origin corresponds to $v$ under this isomorphism. Similarly, each face $F$ of $G$ corresponds to a set $F^{I}$ of standard regions. Each edge $e$ of $G$ corresponds to a geodesic edge $e^{I}$ on the unit sphere.

Now we give an interpretation $I$ to the linear-programming variables at a decomposition star $D$. As usual, we add a superscript $I$ to a variable to indicate its interpretation. Let $\alpha[v, F]^{I}$ be the sum of the interior angles at $v^{I}$ of the metric graph in the standard regions $F^{I}$. Let $y[v]^{I}$ be the length $\left|v^{I}\right|$ of the vertex $v^{I} \in U(D)$ corresponding to $v$. Let $e[v, w]^{I}$ be the length $\left|v^{I}-w^{I}\right|$ of the edge between $v^{I}$ and $w^{I} \in U(D)$. Let

$$
\begin{aligned}
\sigma[F]^{I} & =\sigma_{F}(D), \\
\operatorname{sol}[F]^{I} & =\operatorname{sol}\left(F^{I}\right), \\
\tau[F]^{I} & =\tau_{F}(D) .
\end{aligned}
$$

The objective function for the optimization problems is

$$
\max : \quad \sum_{F \in \mathrm{FACE}} \sigma[F] .
$$

Its interpretation under $I$ is the score $\sigma(D)$.

We can write a number of linear inequalities that will be satisfied under our interpretation. For example, we have the bounds

$$
\begin{aligned}
0 & \leq y[v] \leq 2 t_{0}, & v & \in \text { VERTEX }, \\
0 & \leq e[v, w] \leq 2 t_{0}, & (v, w) & \in \text { EDGE } \\
0 & \leq \alpha[v, F] \leq 2 \pi, & (v, F) & \in \text { ANGLE } \\
0 & \leq \operatorname{sol}[F] \leq 4 \pi & F & \in \text { FACE }
\end{aligned}
$$

There are other linear relations that are suggested directly by the definitions or the geometry. Here, $v$ belongs to VERTEX:

$$
\begin{aligned}
\tau[F] & =\operatorname{sol}[F] \zeta p t-\sigma[F], \\
2 \pi & =\sum_{F: v \in F} \alpha[v, F], \\
\operatorname{sol}[F] & =\sum_{v \in F} \alpha[v, F]-(\operatorname{len}(F)-2) \pi .
\end{aligned}
$$

There are long lists of additional inequalities that come from interval arithmetic verifications. Many are specifically designed to give relations between the variables.

$$
\begin{array}{ccc}
\sigma[F], & \tau[F], & \alpha[v, F], \\
\operatorname{sol}[F], & y[v], & e[v, w],
\end{array}
$$


whenever $F^{I}$ is a single standard region having three sides. Similarly, other computer calculations give inequalities for $\sigma[F]$ and related variables, when the length of $F$ is 4 . A complete list of inequalities that are used for triangular and quadrilateral faces is found in [Ha16].

For exceptional faces, we have an admissible weight function $w(F)$. According to definitions $w(F)=\tau[F] / p t$, so that the inequalities for the weight function can be expressed in terms of the linear program variables.

When the exceptional face is not an aggregate, then it also satisfies the inequalities of Lemma 20.2.

\subsection{Error Analysis}

The variables of the linear programming problem are the dihedral angles, the scores of each of the standard clusters, and their edge lengths.

We subject these variables to a system of linear inequalities. First, the dihedral angles around each vertex sum to $2 \pi$. The dihedral angles, solid angles, and score are related by various linear inequalities as described in Section 23.3. The solid-angle variables are linear functions of dihedral angles. We have

$$
\sigma(D)=\sigma_{S_{1}}(D)+\cdots+\sigma_{S_{p}}(D)+\sigma_{R_{1}}(D)+\cdots+\sigma_{R_{q}}(D) .
$$

Forgetting the origin of the scores, solid angles, and dihedral angles as nonlinear functions of the standard clusters and treating them as formal variables subject only to the given linear inequalities, we obtain a linear programming bound on the score.

Floating-point arithmetic was used freely in obtaining these bounds. The linear programming package CPLEX was used (see www.cplex.com). However, the results, once obtained, could be checked rigorously as follows. ${ }^{136}$

We present an informal analysis of the floating-point errors. For each quasi-regular tetrahedron $S_{i}$ we have a nonnegative variable $x_{i}=p t-\sigma\left(S_{i}\right)$. For each quad cluster $R_{k}$, we have a nonnegative variable $x_{k}=-\sigma\left(R_{k}\right)$. A bound on $\sigma(D)$ is $p t-\sum_{i \in I} x_{i}$, where $p$ is the number of triangular standard regions, and $I$ indexes the faces of the plane graph. We give error bounds for a linear program involving scores and dihedral angles. Similar estimates can be made if there are edges representing edge lengths. Let the dihedral angles be $x_{j}$, for $j$ in some indexing set $J$. Write the linear constraints as $A x \leq b$. We wish to maximize $c \cdot x$ subject to these constraints, where $c_{i}=-1$, for $i \in I$, and $c_{j}=0$, for $j \in J$. Let $z$ be an approximate solution to the inequalities $z A \geq c$ and $z \geq 0$ obtained by numerical methods. Replacing the negative entries of $z$ by 0 we may assume that $z \geq 0$ and that $z A_{i}>c_{i}-\varepsilon$, for $i \in I \cup J$, and some small error $\varepsilon$. If we obtain the numerical bound $p p t+z \cdot b<7.9999 p t$, and if $\varepsilon<10^{-8}$, then $\sigma(D)$ is less than 8 pt. In fact, we note that

$$
\left(\frac{z}{1+\varepsilon}\right) A_{i}
$$

\footnotetext{
136 The output from each linear program that has no exceptional regions has been double checked with interval arithmetic. Predictably, the error bounds presented here were satisfactory (1/2002).
} 
is at least $c_{i}$ for $i \in I$ (since $c_{i}=-1$ ), and that it is greater than $c_{i}-\varepsilon /(1+\varepsilon)$, for $i \in J$ (since $\left.c_{i}=0\right)$. Thus, if $N \leq 60$ is the number of vertices, and $p \leq 2(N-2) \leq 116$ is the number of triangular faces,

$$
\begin{aligned}
\sigma(D) & \leq p p t+c \cdot x \leq p p t+\left(\frac{z}{1+\varepsilon}\right) A x+\frac{\varepsilon}{1+\varepsilon} \sum_{j \in J} x_{j} \\
& \leq p p t+\frac{z \cdot b}{1+\varepsilon}+\frac{\varepsilon}{1+\varepsilon} 2 \pi N \\
& \leq \frac{p p t+z \cdot b+\varepsilon(p p t+2 \pi N)}{(1+\varepsilon)} \\
& \leq \frac{7.9999 p t+10^{-8}(116 p t+500)}{1+10^{-8}}<8 p t .
\end{aligned}
$$

In practice, we used $0.4429<0.79984 p t$ as our cutoff, and $N \leq 14$ in the interesting cases, so that much tighter error estimates are possible.

\section{Elimination of Aggregates}

The proof of the following theorem occupies the entire section. It eliminates all the pathological cases that we have had to carry along until now.

Theorem 24.1. Let $D$ be a contravening decomposition star, and let $G$ be its tame graph. Every face of $G$ corresponds to exactly one standard region of $D$. No standard region of $D$ has any enclosed vertices from $U(D)$. (That is, a decomposition star with one of the aggregates shown in Fig. 20.1 is not contravening.)

\subsection{Triangle and Quad Branching}

Section 25 discusses branch and bound strategies. Branch and bound strategies replace a single linear program with a series of linear program, when a single linear program does not suffice. There is one case of branch and bound that we need before Section 25 . This is a branching on triangular and quadrilateral faces.

We divide triangular faces with corners $v_{1}, v_{2}, v_{3}$ into two cases:

$$
\begin{aligned}
& e\left[v_{1}, v_{2}\right]+e\left[v_{2}, v_{3}\right]+e\left[v_{3}, v_{1}\right] \leq 6.25, \\
& e\left[v_{1}, v_{2}\right]+e\left[v_{2}, v_{3}\right]+e\left[v_{3}, v_{1}\right] \geq 6.25,
\end{aligned}
$$

whenever sufficiently good bounds are not obtained as a single linear program. We also divide quadrilateral faces into four cases: two flat quarters, two flat quarters with a diagonal running in the other direction, four upright quarters forming a quartered octahedron, and the mixed case. (A mixed case by definition is any case that is not one of the other three.) In general, if there are $r_{1}$ triangles and $r_{2}$ quadrilaterals, we obtain as many as $2^{r_{1}+2 r_{2}}$ cases by breaking the various triangles and quadrilaterals into subcases.

We break triangular faces and quadrilaterals into subcases, as needed in the linear programs that follow, without further comment. 


\subsection{A Pentagonal Hull with $n=8$}

The next few sections treat the nonpolygonal standard regions described in Remark 20.18. In this subsection there is an aggregate of the octagonal region and a triangle has a pentagonal hull. Let $P$ denote this aggregate.

Lemma 24.2. Let G be a contravening plane graph with the aggregate of Remark 20.18. Some vertex on the pentagonal face has type not equal to $(3,0,1)$.

Proof. If every vertex on the pentagonal face has type $(3,0,1)$, then at the vertex of the pentagon meeting the aggregated triangle, the four triangles together with the octagon give

$$
t_{8}+\sum_{(4)} \tau_{\mathrm{LP}}(4,0,2 \pi-2(1.153))>(4 \pi \zeta-8) p t,
$$

so that the graph does not contravene.

For a general contravening plane graph with this aggregate, we have bounds

$$
\begin{aligned}
& \sigma_{F}(D) \leq p t+s_{8}, \\
& \tau_{F}(D) \geq t_{8} .
\end{aligned}
$$

We add the inequalities $\tau[F]>t_{8}$ and $\sigma[F]<p t+s_{8}$ to the exceptional face. There is no other exceptional face, because $t_{8}+t_{5}>(4 \pi \zeta-8) p t$. We run the linear programs for all tame graphs with the property asserted by Lemma 24.2. Every upper bound is less than $8 \mathrm{pt}$, so that there are no contravening decomposition stars with this configuration.

\section{3. $n=8$, Hexagonal Hull}

We treat the two cases from Remark 20.18 that have a hexagonal hull (Fig. 20.1). One can be described as a hexagonal region with an enclosed vertex that has height at most $2 t_{0}$ and distance at least $2 t_{0}$ from each corner over the hexagon. The other is described as a hexagonal region with an enclosed vertex of height at most $2 t_{0}$, but this time with distance less than $2 t_{0}$ from one of the corners over the hexagon.

The argument for the case $n=8$ with a hexagonal hull is similar to the argument of Section 24.2. Add the inequalities $\tau[R]>t_{8}$ and $\sigma[R]<s_{8}$ for each hexagonal region. Run the linear programs for all tame graphs, and check that these additional inequalities yield linear programming bounds under $8 \mathrm{pt}$.

\section{4. $n=7$, Pentagonal Hull}

We treat the two cases illustrated in Fig. 20.1 that have a pentagonal hull. These cases require more work. One can be described as a pentagon with an enclosed vertex that has height at most $2 t_{0}$ and distance at least $2 t_{0}$ from each corner of the pentagon. The other 
is described as a pentagon with an enclosed vertex of height at most $2 t_{0}$, but this time with distance less than $2 t_{0}$ from one of the corners of the pentagon.

In discussing various maps, we let $v_{i}$ be the corners of the regions, and we set $y_{i}=\left|v_{i}\right|$ and $y_{i j}=\left|v_{i}-v_{j}\right|$. The subscript $F$ is dropped, when there is no great danger of ambiguity.

Add the inequalities $\tau[F]>t_{7}, \sigma[F]<s_{7}$ for the pentagonal face. There is no other exceptional region, because $t_{5}+t_{7}>(4 \pi \zeta-8) p t$. With these changes, of all the tame plane graphs with a pentagonal face and no other exceptional face, all but one of the linear programs give a bound under $8 \mathrm{pt}$.

The plane graph $G_{0}$ that remains is easy to describe. It is the plane graph with eleven vertices, obtained by removing from an icosahedron a vertex and all five edges that meet at that vertex.

We treat the case $G_{0}$. Let $v_{12}$ be the vertex enclosed over the pentagon. We let $v_{1}, \ldots, v_{5}$ be the five corners of $U(D)$ over the pentagon. Break the pentagon into five simplices along $\left\{0, v_{12}\right\}: S_{i}=\left\{0, v_{12}, v_{i}, v_{i+1}\right\}$. We have LP-derived bounds (in the sense of Remark 23.4) $y\left[v_{i}\right] \leq 2.168$, and $\alpha\left[v_{i}, F\right] \leq 2.89$, for $i=1,2,3,4$, 5. In particular, the pentagonal region is convex, for every contravening star $D \in \operatorname{DS}\left(G_{0}\right)$.

Further LP-derived inequalities are

$$
\sigma[F]>-0.2345 \text { and } \tau[F]<0.644 .
$$

By using branch and bound arguments on the triangular faces, as described in Section 24.1, we can improve the LP-derived inequality to

$$
\tau[F]<0.6079 .
$$

Another LP-derived inequality gives a bound on the perimeter:

$$
\sum\left|v_{i}-v_{i+1}\right| \leq 11.407 .
$$

Yet another LP-derived inequality states that if $v_{1}, v_{2}, v_{3}$ are consecutive corners over the pentagonal region, then

$$
\left|v_{1}-v_{2}\right|+\left|v_{2}-v_{3}\right|<4.804 .
$$

Lemma 24.3. Assume that $R$ is a pentagonal standard region with an enclosed vertex $v$ of height at most $2 t_{0}$. Assume further that

- $\left|v_{i}\right| \leq 2.168$ for each of the five corners.

- Each interior angle of the pentagon is at most 2.89.

- If $v_{1}, v_{2}, v_{3}$ are consecutive corners over the pentagonal region, then $\left|v_{1}-v_{2}\right|+$ $\left|v_{2}-v_{3}\right|<4.804$.

- $\sum_{5}\left|v_{i}-v_{i+1}\right| \leq 11.407$.

Then $\sigma_{R}(D)<-0.2345$ or $\tau_{R}(D)>0.6079$.

Proof. This is Lemma 14.5.

Since the bound $\tau_{R}(D)>0.6079$ contradicts the LP-derived inequality $\tau[F]<$ 0.6079 , this case does not occur in a contravening graph. 


\subsection{Type $(p, q, r)=(5,0,1)$}

We return briefly to the case of six standard regions around a vertex discussed in Remark 20.19. In the plane graph they are aggregated into an octagon. We take each of the remaining cases with an octagon, and replace the octagon with a pentagon and six triangles around a new vertex. There are eight ways of doing this. All eight ways in each of the cases gives an LP bound under $8 \mathrm{pt}$. This completes this case.

The second aggregate shown in Fig. 20.4 contains a pentagon-triangle combination that was ruled out by Lemma 21.10.

\subsection{Summary}

Lemma 24.4. None of the aggregates of Remarks 20.19 and 20.18 appears in a contravening star. In particular, all regions are bounded by simple polygons, and each face of the graph $G(D)$ corresponds to exactly one standard region.

Proof. The proof is the main result of this section.

\section{Branch and Bound Strategies}

When a single linear program does not give sufficiently good bounds, we apply branch and bound methods to improve the bound. By branching repeatedly, we are able to show in every case that a given tame graph is not contravening.

By relying to a greater degree on results that appear in unpublished (but publicly available) computer logs, this section is more technical than the others. The purpose of the section is to give a sketch of the various ways that the various decomposition stars are divided into cases according to a branch and bound strategy.

The first branching strategy has already been described in Section 24.1. It divides the decomposition stars with a given graph into subcases according to the structural properties of triangular and quadrilateral standard regions.

We assume the results from Section 24 that eliminate the most unpleasant types of configurations.

\subsection{Review of Internal Structures}

For the past several sections, it has not been necessary to refer to the internal structure of the standard clusters. This section is different. To describe the branching operations, it is necessary to use details about the structure of standard clusters.

Recall that a quarter is a set of four vertices with five edges of length at least 2 and at most $2 t_{0}$ and a sixth edge of length at least $2 t_{0}$ and at most $2 \sqrt{2}$. The long edge of the quarter is called its diagonal. A set of quarters with pairwise disjoint interiors has been selected. Quarters in this set are said to belong to the $Q$-system. The $Q$-system has been constructed in such a way that if one quarter along a diagonal lies in the $Q$-system, 
then all quarters along that diagonal lie in the $Q$-system. An anchor is a vertex of the packing that has distance at least 2 and at most $2 t_{0}$ from both endpoints of a diagonal. Each diagonal has a context $(n, k)$, with $n \geq k$, where $n$ is the number of anchors around the diagonal and $n-k$ is the number of quarters that have that diagonal as an edge. If a diagonal has context $(n, k)$, then $k$ is the number of gaps that occur between anchors; that is, spaces that are not filled in by quarters. The context of a quarter is defined to be the context of its diagonal.

Recall that a quarter (or its diagonal) is said to be upright if one endpoint of its diagonal is the origin. A quarter is said to be flat if it is not upright and if some vertex of the quarter is the origin.

There is a process of simplification of the decomposition stars and their scoring functions that eliminates ${ }^{137}$ many of the contexts $(n, k)$. (The upright quarters are said to be erased.) We assume in the following discussion and lemmas that this procedure has been carried out.

An upright diagonal is said to be a loop when there is a reasonable scheme of inserting a simplex into each gap so that the diagonal is completely surrounded by quarters and the inserted simplices. The simplices that are inserted in the gaps are called anchored simplices. They are constructed in such a way that every edge of an anchored simplex has length at most 3.2. All simplices in a given loop lie over a single standard region. If the gaps cannot be filled with anchored simplices, the upright diagonal is not a loop. Details of this construction can be found in Section 11.5.

In every case the simplices around a given upright diagonal lie in the cone over a single standard region.

Lemma 25.1. Consider an upright diagonal that is a loop. Let $R$ be the standard region that contains the upright diagonal and its surrounding simplices. Then the following contexts $(n, k)$ are the only ones possible. Moreover, the constants that appear in the columns marked $\sigma$ and $\tau$ are upper and lower bounds respectively for $\sigma_{R}(D)$ and $\tau_{R}(D)$ when $R$ contains one loop of that context.

\begin{tabular}{lccl}
\hline Std. region & $(n, k)$ & $\sigma$ & \multicolumn{1}{c}{$\tau$} \\
\hline$R$ quad & $(4,0)$ & -0.0536 & 0.1362 \\
$R$ pentagon & $(4,1)$ & $s_{5}$ & 0.27385 \\
& $(5,0)$ & -0.157 & 0.3665 \\
$R$ hexagon & $(4,1)$ & $s_{6}$ & 0.41328 \\
& $(4,2)$ & -0.1999 & 0.5309 \\
$R$ heptagon & $(5,1)$ & -0.37595 & 0.65995 \\
& $(4,1)$ & $s_{7}$ & 0.55271 \\
$R$ octagon & $(4,1)$ & -0.25694 & 0.67033 \\
& $(4,2)$ & -0.31398 & 0.60722 \\
& & & \\
\hline
\end{tabular}

Proof. This is Lemma 13.5 .

\footnotetext{
${ }^{137}$ In detail, we assume that all the contexts that do not carry a penalty have been erased. We leave loops, 3-crowded, 4-crowded, and 3-unconfined upright diagonals unerased at this point.
} 


\subsection{3-Crowded and 4-Crowded Upright Diagonals}

Definition 25.2. Consider an upright diagonal that is not a loop. Let $R$ be the standard region that contains the upright diagonal and its surrounding quarters. Then the contexts $(4,1)$ and $(5,1)$ are the only contexts possible. In the context $(4,1)$, if there does not exist a plane through the upright diagonal such that all three quarters lie in the same halfspace bounded by the plane, then we say that the context is 3-unconfined. If such a plane exists, then we say that the context is 3 -crowded. We call the context $(5,1)$ a 4 -crowded upright diagonal. Thus, every upright diagonal is exactly one of the following: a loop, 3-unconfined, 3-crowded, or 4-crowded. A contravening decomposition star contains at most one upright diagonal that is 3 -crowded or 4 -crowded. See Section 11.9 for a proof of these facts and for further details.

Lemma 25.3. Let $R$ be a standard region that contains an upright diagonal that is 4-crowded. Then

$$
\sigma_{R}(D)<-0.25 \text { and } \tau_{R}(D)>0.4
$$

Let $R$ be a standard region that contains an upright diagonal that is 3-crowded. Then

$$
\sigma_{R}(D)<-0.4339 \text { and } \tau_{R}(D)>0.5606
$$

Proof. See Lemmas 11.11 and 11.18.

Lemma 25.4. A contravening decomposition star does not contain any upright diagonals that are 3-crowded.

Proof. If we have an upright diagonal that is 3-crowded, then there is only one exceptional region $\left(0.5606+t_{5}>(4 \pi \zeta-8) p t\right)$. We add the inequalities $\tau>0.5606$ and $\sigma<-0.4339$ to the exceptional region. All linear programming bounds drop under $8 \mathrm{pt}$ when these changes are made.

Upright diagonals that are 4-crowded require more work. We begin with a lemma.

Lemma 25.5. Let $\alpha$ be the dihedral angle along the large gap along an upright diagonal that is 4-crowded. Let $F$ be the union of the four upright quarters along the upright diagonal. Let $v_{1}$ and $v_{2}$ be the anchors of $U(D)$ lying along the large gap. If $\left|v_{1}\right|+\left|v_{2}\right|<4.6$, then $\alpha>1.78$ and $\sigma_{F}(D)<-0.31547$.

Proof. The bound $\alpha>1.78$ comes from the inequality archive. ${ }^{138}$ The upper bound on the score is a linear programming calculation involving the inequality $\alpha>1.78$ and the known inequalities on the score of an upright quarter.

\footnotetext{
${ }^{138}$ CALC-161665083.
} 
Lemma 25.6. A contravening decomposition star does not contain any upright diagonals that are 4-crowded.

Proof. Add the inequalities $\sigma_{R}(D)<-0.25$ and $\tau_{R}(D)>0.4$ at the exceptional regions. An upright diagonal that is 4-crowded does not appear in a pentagon for purely geometrical reasons. Run the linear programs for all tame plane graphs with an exceptional region that is not a pentagon. If this linear program fails to produce a bound of $8 \mathrm{pt}$, we use the lemma to branch into two cases: either $y\left[v_{1}\right]+y\left[v_{2}\right] \geq 4.6$ or $\sigma[R]<-0.31547$. In every case the bound drops below $8 \mathrm{pt}$.

\subsection{Five Anchors}

Now turn to the decomposition stars with an upright diagonal with five anchors. Five quarters around a common upright diagonal in a pentagonal region can certainly occur. We claim that any other upright diagonal with five anchors leads to a decomposition star that does not contravene. In fact, the only other possible context is $(n, k)=(5,1)$ (see Lemma 25.1).

Lemma 25.7. Let $D$ be a contravening decomposition star. Then there are no loops with context $(5,1)$ in $D$.

Proof. By Lemma 25.1, the standard region $R$ that contains the loop must be a hexagon. By the same lemma, we have

$$
\tau_{R}(D)>0.65995 \text { and } \sigma_{R}(D)<-0.37595 \text {. }
$$

Add these constraints to the linear program of the tame graphs with a hexagonal face. The LP-bound on $\sigma(D)$ with these additional inequalities is less than $8 \mathrm{pt}$.

\subsection{Penalties}

From now on, we assume that there are no loops with context $(5,1)$, and no 3 -crowded or 4-crowded upright diagonals. This leaves various loops and 3-unconfined upright diagonals.

At times, it is necessary to erase certain loops and 3-unconfined upright diagonals. There is a penalty for doing so. Let $D$ be a decomposition star with an upright diagonal $\{0, v\}$. Let $D^{\prime}$ be the decomposition star that is identical in all respects, except that $v$ and all indices in the decomposition star that point to $v$ (in the sense of Section 6.1) have been deleted. Let $R$ be the standard region of $D$ over which $v$ is located, and let $R^{\prime}$ be the corresponding standard region of $D^{\prime}$. We say that the upright diagonal can be erased with penalty $\pi_{R}$ if

$$
\sigma_{R}(D) \leq \sigma_{R^{\prime}}\left(D^{\prime}\right)+\pi_{R}
$$


Definition 25.8. When we break a single region into smaller regions (by taking the part of the region that meets the cone over a quarter, anchored simplex, and so forth) the smaller regions will be called subregions. An anchored simplex that overlaps a flat quarter is said to mask the flat quarter. (Masked flat quarters are not in the $Q$-system.)

Remark 25.9. A function $\hat{\sigma}$ has been defined in Section 11.10. The details of the definition of this function are not important here. It is proved there that $\hat{\sigma}$ is a good upper bound on the scoring function on flat quarters no matter what the origin of the flat quarter. It gives bounds for flat quarters in the $Q$-system, masked quarters, isolated quarters, and all the other types of flat quarters. The function $\hat{\tau}$ on the space of flat quarters is defined as

$$
\hat{\tau}(Q)=\operatorname{sol}(Q) \zeta p t-\hat{\sigma}(Q) .
$$

Remark 25.10. At times, we work with various upper bounds to $\sigma_{R}(D)$, say,

$$
\sigma_{R}(D) \leq f_{R}(D) .
$$

When we have a specific upper bound $f_{R}(D)$ in view, then we will also say that the upright diagonal can be erased with penalty $\pi_{R}$ if

$$
f_{R}(D) \leq f_{R^{\prime}}\left(D^{\prime}\right)+\pi_{R} .
$$

In more detail, let $R=\left\{R_{1}, \ldots, R_{k}\right\}$ be the set of subregions over the anchored simplices in a loop. Let $f_{R_{i}}(D)$ be the approximations of the score of each anchored simplex. Let $Q_{1}, \ldots, Q_{\ell}$ be the flat quarters masked by the anchored simplices in the loop. Let $R^{\prime}$ be the subregion of points in the union of $R$ that are not in the cone over any $Q_{i}$. Then we erase with penalty $\pi_{R}$ if

$$
\sum_{i} f_{R_{i}}(D) \leq \sum_{\ell} \hat{\sigma}\left(Q_{j}\right)=\operatorname{vor}_{R^{\prime}, 0}(D)+\pi_{R} .
$$

If the upright diagonal is not a loop, we include in the set $R$ all regions along the "gaps" around the upright diagonal.

Sections 13.4 and 13.6 makes various estimates of the penalties that are involved in erasing various loops and 3-unconfined upright diagonals. Most of the penalties are calculated as integer combinations of the constants $\xi_{\Gamma}=0.01561, \xi_{V}=0.003521$, and 0.008. It is proved ${ }^{139}$ in Section 11.7 that $\xi_{\Gamma}$ is the penalty for erasing a single upright quarter of compression type, and that $\xi_{V}$ is the penalty for erasing a single upright quarter of Voronoi type.

Lemma 25.11. Let $\{0, v\}$ be an upright diagonal.

- If the upright diagonal is 3-unconfined, then the upright diagonal can be erased with penalty 0.008 .

${ }^{139}$ CALC-751772680 and CALC-310679005. 
- If the upright diagonal is 3-unconfined and it masks a flat quarter, then the upright diagonal can be erased with penalty 0.

- If a flat quarter is masked, then its diagonal has length at least 2.6. Also, if the diagonal of a masked flat quarter has length at most 2.7, then the height of its central vertex is at least 2.2.

Proof. See Section 11.9.

\subsection{Pent and Hex Branching}

If a single linear program does not yield the bound $\sigma(D)<8 \mathrm{pt}$, then we divide the set of decomposition stars with graph $G$ into several subsets, according to the arrangements of quarters inside each standard cluster. This section gives a rough classification of possible arrangements of quarters in the cone over pentagonal and hexagonal standard regions.

The possibilities are listed in Figs. 25.1 and 25.2 only up to symmetry by the dihedral group action on the polygon. We do not prove the completeness of the list, but its completeness can be seen by inspection, in view of the comments that follow here and in Section 25.4. Details about the size of the penalties can be found in Section 13.6.

The conventions for generating the possibilities are different for the pentagons and hexagons than for the heptagons and octagons. We describe the pentagons and hexagons first. We erase all 3-unconfined upright diagonals. If there is one loop we leave the loop in the figure. If there are two loops (so that both necessarily have context $(n, k)=(4,1)$ ), we erase one and keep the other.

The figures are interpreted as follows. An internal vertex in the polygon represents an upright diagonal. Edges from that vertex are in 1-1 correspondence with the anchors around that upright diagonal. Edges between nonadjacent vertices of the polygon represent the diagonals of flat quarters. We draw all edges from an upright diagonal to its anchors, and all edges of length $\left[2 t_{0}, 2 \sqrt{2}\right]$ that are not masked by upright quarters. Since the only remaining upright quarters belong to loops, the four simplices around a loop are anchored simplices and the edge opposite the diagonal has length at most 3.2.

Various inequalities in the inequality archive have been designed for subregions of pentagons. Additional inequalities have been designed for subregions in hexagonal regions. Thus, we are able to obtain greatly improved linear programming bounds when

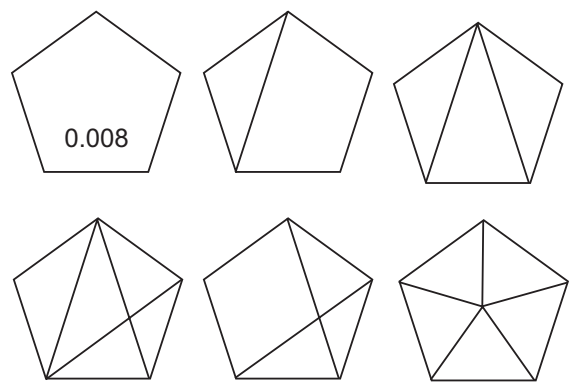

Fig. 25.1. Pentagonal face refinements. 


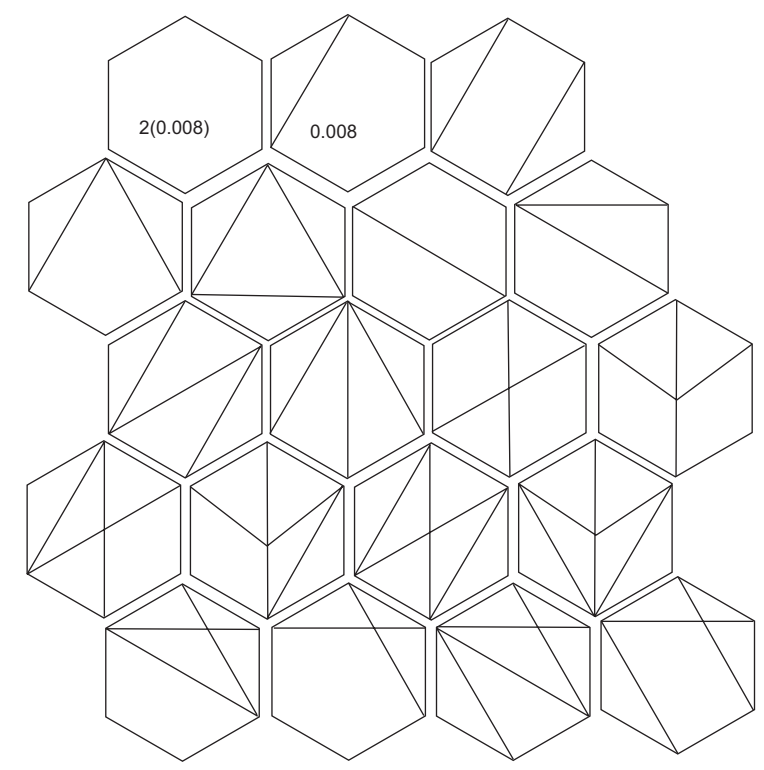

Fig. 25.2. Hexagonal face refinements. The only figures with a penalty are the first two on the top row and those on the bottom row. The first two on the top row have penalties 2(0.008) and 0.008 . Those on the bottom row have penalties $3 \xi_{\Gamma}, 3 \xi_{\Gamma}, \xi_{\Gamma}+2 \xi_{V}$, and $\xi_{\Gamma}+2 \xi_{V}$.

we break each pentagonal region into various cases, according to the list of Figs. 25.1 and 25.2.

\subsection{Hept and Oct Branching}

When the figure is a heptagon or octagon, we proceed differently. We erase all 3unconfined upright diagonals and all loops (either context $(n, k)=(4,1)$ or $(4,2)$ ) and draw only the flat quarters. An undrawn diagonal of the polygon has length at least $2 t_{0}$. Overall, in these cases much less internal structure is represented.

In the cases where 3-confined upright diagonals or loops have been erased, a number indicating a penalty accompanies the diagram (Figs. 25.3 and 25.4). These penalties are derived in Sections 13.6 and 13.4.

Define values

$$
Z(3,1)=0.00005 \text { and } \quad D(3,1)=0.06585 \text {. }
$$

Here are some special arguments that are used for heptagons and octagons.

25.6.1. One Flat Quarter. Suppose that the standard region breaks into two subregions: the triangular region of a flat quarter $Q$ and one other. Let $n=n(R) \in\{7,8\}$. We have the inequality

$$
\sigma_{R}(D)<(\hat{\sigma}(Q)-Z(3,1))+s_{n}+\xi_{\Gamma}+2 \xi_{V} .
$$




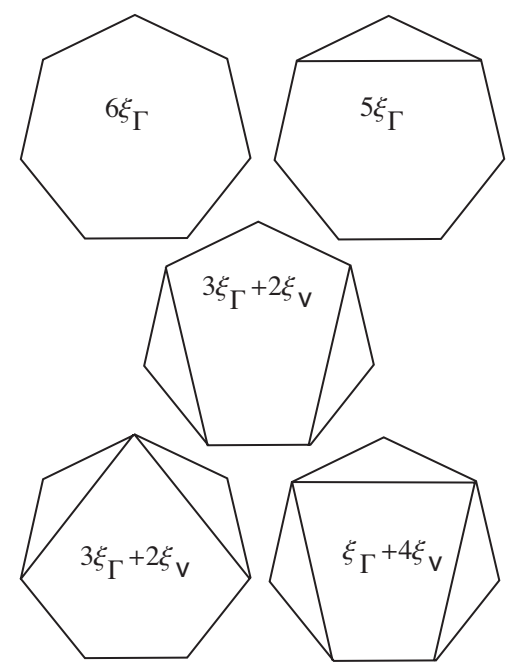

Fig. 25.3. Heptagonal face refinements.

The penalty term $\xi_{\Gamma}+2 \xi_{V}$ comes from a possible anchored simplex masking a flat quarter. Let $v$ be the central vertex of the flat quarter $Q$. Let $\left\{v_{1}, v_{2}\right\}$ be its diagonal. Masked flat quarters satisfy restrictive edge constraints. It follows from Section 11.10 that we have one of the following three possibilities:

1. $y[v] \geq 2.2$.

2. $e\left[v_{1}, v_{2}\right] \geq 2.7$.

3. $\sigma_{R}(D)<(\hat{\sigma}(Q)-Z(3,1))+s_{n(R)}$.

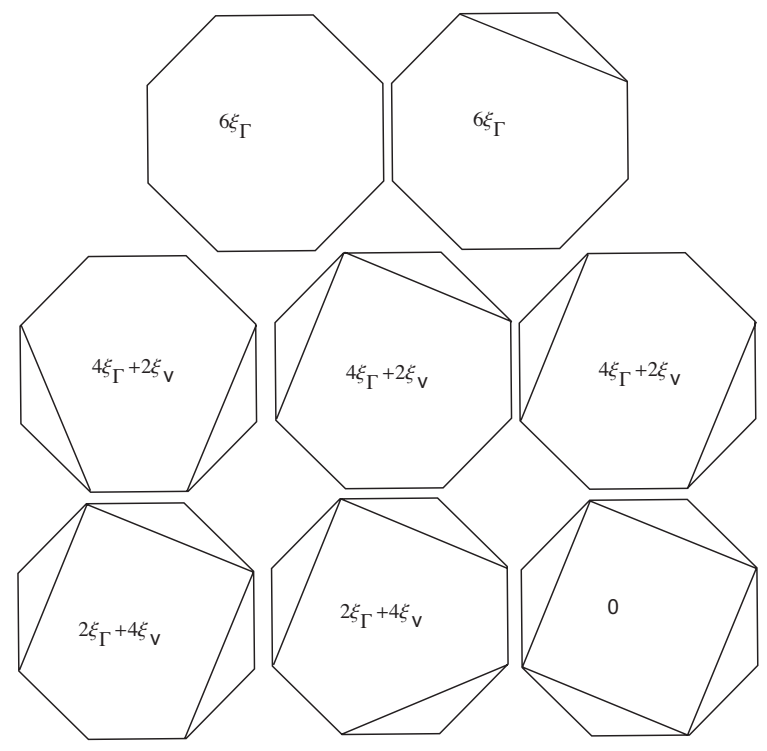

Fig. 25.4. Octagonal face refinements. 
25.6.2. Two Flat Quarters. We proceed similarly if the standard region $R$ breaks into three subregions: two regions $R_{1}$ and $R_{2}$ cut out by flat quarters $Q_{1}, Q_{2}$ and one other region made from what remains. Write $\hat{\sigma}_{1}$ for $\hat{\sigma}\left(Q_{1}\right)$, and so forth. It follows from Section 11.10 that we have one of the following three possibilities:

1. The height of a central vertex is at least 2.2.

2. The diagonal of a flat quarter is at least 2.7.

3.

$$
\begin{aligned}
\sigma_{R}(D)<\left(\hat{\sigma}_{1}-Z(3,1)\right)+\left(\hat{\sigma}_{2}-Z(3,1)\right)+s_{n(R)}, \\
\tau_{R}(D)>\left(\hat{\tau}_{1}-D(3,1)\right)+\left(\hat{\tau}_{2}-D(3,1)\right)+t_{n(R)} .
\end{aligned}
$$

With heptagons, it is helpful on occasion to use an upper bound on the penalty of $3 \xi_{\Gamma}=0.04683$. This bound holds if neither flat quarter is masked by a loop. For this, it suffices to show that the first two of the given three cases do not hold.

If there is a loop of context $(n, k)=(4,2)$, we have the upper bounds of Lemma 25.1. If, on the other hand, there is no loop of context $(n, k)=(4,2)$, then we have the upper bound

$$
\sigma_{R}(D) \leq\left(\hat{\sigma}\left(Q_{1}\right)-Z(3,1)\right)+\left(\hat{\sigma}\left(Q_{2}\right)-Z(3,1)\right)+s_{n(R)}+2\left(\xi_{\Gamma}+2 \xi_{V}\right),
$$

where $n(R) \in\{7,8\}$.

\subsection{Branching on Upright Diagonals}

We divide the upright simplices into two domains depending on the height of the upright diagonal, using $|v|=2.696$ as the dividing point. We break the upright diagonals (of unerased quarters in the $Q$-system) into cases:

1. The upright diagonal has height at most 2.696.

2. The upright diagonal $\{0, v\}$ has height at least 2.696, and some anchor $w$ along the flat quarter satisfies $|w| \geq 2.45$ or $|v-w| \geq 2.45$. (There is a separate case here for each anchor $w$.)

3. The upright diagonal $\{0, v\}$ has height at least 2.696, and every anchor $w$ along the flat quarter satisfies $|w| \leq 2.45$ and $|v-w| \leq 2.45$.

Many inequalities have been specially designed to hold on these smaller domains. They are included into the linear programming problems as appropriate.

When all the upright quarters can be erased, then the case for upright quarters follows from some other case without the upright quarters. An upright quarter can be erased in the following situations. If the upright quarter $Q$ has compression type (in the sense of Definition 7.8) and the diagonal has height at least 2.696, then ${ }^{140}$

$$
\sigma(Q)<\operatorname{s-vor}_{0}(Q)
$$

${ }^{140}$ CALC-214637273. 
(If there are masked flat quarters, they become scored by $\hat{\sigma}$.) If an upright quarter has Voronoi type and the anchors $w$ satisfy $|w| \leq 2.45$ and $|v-w| \leq 2.45$, then the quarter can be erased ${ }^{141}$

$$
\sigma(Q)<\mathrm{s}^{-\operatorname{vor}_{0}}(Q) .
$$

In general, we only have the weaker inequality ${ }^{142}$

$$
\sigma(Q)<\text { s-vor }_{0}(Q)+0.003521 .
$$

In a pentagon or hexagon, consider an upright diagonal with three upright quarters, that is, context $(n, k)=(4,1)$. If the upright diagonal has height at most 2.696, and if an upright quarter shares both faces along the upright diagonal with other upright quarters, then we may assume that the upright quarter has compression type. For otherwise, there is a face of circumradius at least $\sqrt{2}$, and hence two upright quarters of Voronoi type. The inequality

$$
\text { octavor }<\text { octavor }_{0}-0.008 \text {, }
$$

if $y_{1} \in\left[2 t_{0}, 2.696\right]$, and $\eta_{126} \geq \sqrt{2}$, shows that the upright quarters can be erased without penalty because

$$
\xi_{\Gamma}-0.008-0.008<0 .
$$

If erased, the case is treated as part of a different case.

This allows the inequalities ${ }^{143}$ to be used that relate specifically to upright quarters of compression type. Furthermore, it can often be concluded that all three upright quarters have compression type. For this, we use various inequalities in the archive which can often be used to show that if the anchored simplex has a face of circumradius at least $\sqrt{2}$, then the linear programming bound on $\sigma(D)$ is less than $8 \mathrm{pt}$.

\subsection{Branching on Flat Quarters}

We make a few general remarks about flat quarters.

Remark 25.12. Information about the internal structure of an exceptional face gives improvements to the constants $1.4 \mathrm{pt}$ and $1.5 \mathrm{pt}$ of Property 4 in the definition of admissible weight assignments. (The bounds remain fixed at $1.4 \mathrm{pt}$ and $1.5 \mathrm{pt}$, but these arguments allow us to specify more precisely which simplices contribute to these bounds.) These constants contribute to the bound on $\tau(D)$ through the admissible weight assignment. Assume that at the vertex $v$ there are four quasi-regular tetrahedra and an exceptional face, and that the exceptional face has a flat quarter with central vertex $v$. The calculations of Section 22.3 show that the union $F$ of the four quasi-regular tetrahedra and exceptional region give $\tau_{F}(D) \geq 1.5 \mathrm{pt}$. If there is no flat quarter with central vertex $v$, then the union $F$ of four quasi-regular tetrahedra along $\{0, v\}$ give $\tau_{F}(D) \geq 1.5 p t$. We can make similar improvements when $\operatorname{tri}(v)=3$.

\footnotetext{
141 CALC-378432183.

142 CALC-310679005.

${ }^{143}$ See, for example, CALC-867513567-*.
} 
Remark 25.13. There are a few other interval-based inequalities that are used in particular cases. The inequalities $y_{1} \leq 2.2, y_{4} \leq 2.7, \eta_{234}, \eta_{456} \leq \sqrt{2}$ imply that the flat quarter has compression type (see Section 7.1). The circumradius is not a linear programming variable, so its upper bound must be deduced from edge-length information.

If all three corners of a flat quarter have height at most 2.14 , and if the diagonal has length less than 2.77 , then the circumradius of the face containing the origin and diagonal is at most $\eta(2.14,2.14,2.77)<\sqrt{2}$. This allows us to branch combine into three cases.

Lemma 25.14. Let $Q$ be a flat quarter whose corners $v_{i}$ have height at most 2.14 and whose diagonal is at most 2.77. Then one of the following is true:

1. $\sigma(Q)=\Gamma(Q)$.

2. The diagonal has length $\leq 2.7, \eta\left(y_{4}, y_{5}, y_{6}\right) \geq \sqrt{2}$, and $\sigma(Q) \leq \mathrm{s}-\operatorname{vor}_{0}(Q)$.

3. The diagonal has length $\geq 2.7$ and $\sigma(Q) \leq \mathrm{s}-\operatorname{vor}_{0}(Q)$.

Proof. Case 1 holds when $Q$ is a quarter of compression type in the $Q$-system. If $Q$ is in the $Q$-system but is not of compression type, then $\eta\left(y_{4}, y_{5}, y_{6}\right) \geq \sqrt{2}$ and $\sigma(Q) \leq$ s-vor $(Q)$. If $Q$ is not in the $Q$-system, then s-vor $(Q)$ is an upper bound Lemma 11.26. If $Q$ is not in the $Q$-system, then its diagonal has length at least 2.7, or the central vertex has height at most 2.2 (see Lemma 25.11.) In this case we use the upper bound s-vor $0(Q)$.

Various inequalities in the archive have been designed specifically for each of these three cases. Thus, whenever the hypotheses of the lemma are met, we are able to improve on the linear programming bounds by breaking into these three cases.

\subsection{Branching on Simplices that Are Not Quarters}

Lemma 25.15. Suppose that a triangular subregion comes from a simplex $S$ with one vertex at the origin and three other vertices of height at most $2 t_{0}$. Suppose that the edge lengths of the fourth, fifth, and sixth edges satisfy $y_{5}, y_{6} \in\left[2 t_{0}, 2 \sqrt{2}\right], y_{4} \in\left[2,2 t_{0}\right]$. Suppose that $\min \left(y_{5}, y_{6}\right) \leq 2.77$. Then one of the following is true:

1. The edges have lengths $y_{5}, y_{6} \in\left[2 t_{0}, 2.77\right], \eta_{456} \geq \sqrt{2}$, and $\sigma(S) \leq \mathrm{s}-\operatorname{vor}_{0}(S)$.

2. $y_{5}, y_{6} \in\left[2 t_{0}, 2.77\right]$, and $\sigma(S) \leq \mathrm{s}-\operatorname{vor}(S)$ (the analytic Voronoi function).

3. An edge $\left(\right.$ say $\left.y_{6}\right)$ has length $y_{6} \geq 2.77$ and $\sigma(S) \leq$ s- $\operatorname{vor}_{0}(S)$.

Proof. If we ignore the statements about $\sigma$, then the conditions in the lemma concerning edge length are exhaustive. The bounds on $\sigma$ in each case are given by Section 9.6.

There are linear programming inequalities that are tailored to each case. 


\subsection{Branching on Quadrilateral Subregions}

One of the inequalities holds for a quadrilateral subregion, if certain conditions are satisfied. One of the conditions is $y_{4} \in[2 \sqrt{2}, 3.0]$, where $y_{4}$ is a diagonal of the subregion. Since this diagonal is not one of the linear programming variables, these bounds cannot be verified directly from the linear program. Instead we use an inequality which relates the desired bound $y_{4} \leq 3$ to the linear programming variables $\alpha[v, F], y_{2}, y_{3}, y_{5}$, and $y_{6}$.

\subsection{Implementation Details for Branching}

We now make a detailed examination of the internal structure of exceptional regions.

A refinement $\tilde{F}$ of a face $F$ of a plane graph $G$ is a set $\tilde{F}$ of faces such that:

1. The intersection of the vertex set of $G$ with that of $\tilde{F}$ is the set $F$.

2. $\tilde{F} \cup\left\{F^{\mathrm{op}}\right\}$ is a plane graph.

We use refinements of faces to describe the internal structure of faces.

We introduce indexing sets FACE $-\tilde{F}$, VERTEX $-\tilde{F}$, ANGLE- $\tilde{F}$, EDGE $-\tilde{F}$, the sets of faces, vertices, angles, and edges in $\tilde{F}$, respectively, analogous to those introduced for $G$.

We create variables $\pi[\tilde{F}]$, and indexed variables

$$
\begin{array}{lll}
\operatorname{sol}\{\mathrm{FACE}-\tilde{F}\}, & \operatorname{sc}\{\mathrm{FACE}-\tilde{F}\} & \tau \operatorname{sc}\{\mathrm{FACE}-\tilde{F}\}, \\
\alpha\{\mathrm{ANGLE}-\tilde{F}\}, & y\{\mathrm{VERTEX}-\tilde{F}\}, & e\{\mathrm{EDGE}-\tilde{F}\} .
\end{array}
$$

(Variables with names " $y[v]$ " and " $e[v, w]$ " were already created for some $v, w \in$ VERTEX $-\tilde{F} \cap$ VERTEX. In these cases we use the variables already created.)

Each vertex $v$ in the refinement will be interpreted either as a vertex $v^{I} \in U(D)$, or as the endpoint of an upright diagonal lying over the standard region $F^{I}$. We interpret the faces of the refinement in terms of the geometry of the decomposition star $D$ variously as flat quarters, upright quarters, anchored simplices, and the other constructs of the fourth paper. This interpretation depends on the context, and is described in greater detail below.

Once the interpretation of faces is fixed, the interpretations are as before for the variable names introduced already: $y, e, \alpha$, sol. The lower and upper bounds for $\alpha$ and sol are as before. The lower and upper bounds for $y[v]$ are 2 and $2 t_{0}$ if $v^{I} \in U(D)$, but if $\left(0, v^{I}\right)$ is an upright diagonal, then the bounds are $\left[2 t_{0}, 2 \sqrt{2}\right]$. The lower and upper bounds for $e$ will depend on the context.

\subsection{Variables Related to Score}

The variables sc are a stand-in for the score $\sigma$ on a face. We do not call them $\sigma$ because the sum of these variables will not in general equal the variable $\sigma[F]$, when $\tilde{F}$ is a refinement of $F$ :

$$
\left[\sum_{F^{\prime} \in \tilde{F}} \operatorname{sc}\left[F^{\prime}\right] \neq \sigma[F]\right] .
$$


We use a weaker relation:

$$
\sigma[F] \leq \sum_{F^{\prime} \in \tilde{F}} \operatorname{sc}\left[F^{\prime}\right]+\pi[\tilde{F}] .
$$

The variable $\pi[\tilde{F}]$ is called the penalty associated with the refinement $\tilde{F}$. (Penalties are discussed at length in Sections 13.6 and 13.4.) The interpretations of sc and $\pi[\tilde{F}]$ are rather involved, and are discussed on a case-by-case basis below. The interpretation of $\tau \mathrm{sc}$ follows from the identity

$$
\tau \operatorname{sc}\left[F^{\prime}\right]=\operatorname{sol}\left[F^{\prime}\right] \zeta p t-\operatorname{sc}\left[F^{\prime}\right], \quad \forall F^{\prime} \in \tilde{F} .
$$

The interpretation of variables that follows might appear to be hodge-podge at first. However, they are obtained in a systematic way. We analyze the proofs and approximations in Paper IV, and define $\mathrm{sc}[F]^{I}$ as the best penalty-free scoring approximation that is consistent with the given face refinement. Here are the details.

If the subregion is a flat quarter, the interpretation of $\operatorname{sc}[F]$ is the function $\hat{\sigma}$, defined in Section 11.10. If the subregion is an upright quarter $Q$, the interpretation of $\operatorname{sc}[F]$ is the function $\sigma(Q)$ from Section 7. If the subregion is an anchored simplex that is not an upright quarter, $\mathrm{sc}[F]$ is interpreted as the analytic Voronoi function vor if the

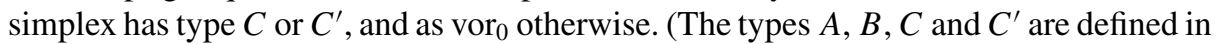
Section 9.4.) Whether or not the simplex has type $C$, the inequality $\operatorname{sc}[F] \leq 0$ is satisfied. In fact, if vor $_{0}$ scoring is used, we note that there are no quoins, and $\varphi\left(1, t_{0}\right)<0$.

If the subregion is triangular, if no vertex represents an upright diagonal, and if the


the simplex has type $A$. In either case, the inequality $\operatorname{sc}[F] \leq$ vor $_{0}$ is satisfied.

In most other cases the interpretation of $\operatorname{sc}[F]$ is vor $_{0}$. However, if $R$ is a heptagon or octagon, and $F$ has four or more sides, then $\operatorname{sc}[F]$ is interpreted as vor ${ }_{0}$ except on simplices of type $A$, where it becomes the analytic Voronoi function.

If $R$ is a pentagon or hexagon, and $F$ is a quadrilateral that is not adjacent to a flat quarter, and if there are no penalties in the region, then the interpretation of $\operatorname{sc}[F]$ is the actual score of the subregion over the subregion. In this case the score $\sigma_{R}$ has a welldefined meaning for the quadrilateral, because it is not possible for an upright quarter in the $Q$-system to straddle the quadrilateral region and an adjacent region. Consequently, any erasing that is done can be associated with the subregion without ambiguity. By the results of Sections 8.4 and 8.5 , we have $\mathrm{sc}[F] \leq 0$. We also have $\mathrm{sc}[F] \leq \operatorname{vor}_{0}$.

One other bound that we have not explicitly mentioned is the bound $\sigma_{R}(D)<s_{n}$. For heptagons and octagons that are not aggregates, this is a better bound than the one used in the definition of tameness (Property 6). In heptagons and octagons that are not aggregates, if we have a subregion with four or more sides, then $\operatorname{sc}[F]<Z(n, k)$ and $\tau \mathrm{sc}[F]>D(n, k)$. (See (13.1) and (13.2) in Section 13.5.)

The variables are subject to a number of compatibility relations that are evident from the underlying definitions and geometry.

$$
\begin{array}{r}
\operatorname{sol}\left[F^{\prime}\right]=\sum_{v \in F^{\prime}} \alpha\left[v, F^{\prime}\right]-\left(\operatorname{len}\left[F^{\prime}\right]-2\right) \pi, \quad \forall F^{\prime}, \\
\sum_{F^{\prime}: v \in F^{\prime}, F^{\prime} \in \mathrm{FACE}-\tilde{F}} \alpha\left[v, F^{\prime}\right]=\alpha[v, F], \quad \forall v .
\end{array}
$$


Assume that a face $F_{1} \in \tilde{F}$ has been interpreted as a subregion $R=F_{1}^{I}$ of a standard region. Assume that each vertex of $F_{1}$ is interpreted as a vertex in $U(D)$ or as the endpoint of an upright diagonal over $F^{I}$. One common interpretation of sc is $\operatorname{vor}_{0, F}(U(D))$, the truncated Voronoi function. When this is the interpretation, we introduce further variables:

$$
\begin{array}{ll}
\operatorname{quo}\left[v, s\left(v, F_{1}\right)\right], & \forall v \in F_{1}, \\
\operatorname{quo}\left[s\left(v, F_{1}\right), v\right], & \forall v \in F_{1}, \\
\operatorname{Adih}\left[v, F_{1}\right], & \forall v \in F_{1} .
\end{array}
$$

We interpret the variables as follows. If $w=s(v)$, and the triangle $\left(0, v^{I}, w^{I}\right)$ has circumradius $\eta$ at most $t_{0}$, then

$$
\begin{aligned}
& \operatorname{quo}[v, w]^{I}=\operatorname{quo}\left(R\left(\left|v^{I}\right| / 2, \eta, t_{0}\right)\right), \\
& \operatorname{quo}[w, v]^{I}=\operatorname{quo}\left(R\left(\left|w^{I}\right| / 2, \eta, t_{0}\right)\right) .
\end{aligned}
$$

If the circumradius is greater than $t_{0}$, we take

$$
\operatorname{quo}[v, w]^{I}=\operatorname{quo}[w, v]^{I}=0 .
$$

The variable Adih has the following interpretation:

$$
\operatorname{Adih}\left[v, F_{1}\right]^{I}= \begin{cases}A\left(\left|v^{I}\right| / 2\right) \alpha\left(v^{I}, F_{1}^{I}\right), & \left|v^{I}\right| \leq 2 t_{0}, \\ 0, & \text { otherwise. }\end{cases}
$$

Under these interpretations, the following identity is satisfied:

$$
\operatorname{sc}\left[F_{1}\right]=\operatorname{sol}\left[F_{1}\right] \varphi_{0}+\sum_{v \in F_{1}} \operatorname{Adih}\left[v, F_{1}\right]-4 \delta_{\text {oct }} \sum \operatorname{quo}[v, w] .
$$

The final sum runs over all pairs $(v, w)$, where $v=s\left(w, F_{1}\right)$ or $w=s\left(v, F_{1}\right)$.

For this to be useful, we need good inequalities governing the individual variables. Such inequalities for $\operatorname{Adih}[v, F]$ and $\operatorname{quo}[v, w]$ are found in Calculations CALC-815275408 and CALC-349475742. To make of use these inequalities, it is necessary to have lower and upper bounds on $\alpha[v, F]$ and $y[v]$. We obtain such bounds as LP-derived inequalities in the sense of Remark 23.4.

\subsection{Appendix: Hexagonal Inequalities}

There are a number of inequalities that have been particularly designed for standard regions that are hexagons. This appendix describes those inequalities. They are generally inequalities involving more than six variables, and because of current technological limitations on interval arithmetic, we were not able to prove these inequalities directly with interval arithmetic.

Instead we give various lemmas that deduce the inequalities from inequalities in a smaller number of variables (small enough to prove by interval arithmetic). 
25.13.1. Statement of Results. There are a number of inequalities that hold in special situations when there is a hexagonal region. Although these inequalities do not appear in the main text of the proof of the Kepler conjecture, they are used in the linear programs.

After stating all of them, we turn to the proofs.

1. If there are no flat quarters and no upright quarters (so that there is a single subregion $F$ ), then

$$
\begin{aligned}
\operatorname{vor}_{0} & <-0.212, \\
\tau_{0} & >0.54525 .
\end{aligned}
$$

2. If there is one flat quarter and no upright quarters, there is a pentagonal subregion $F$. It satisfies

$$
\begin{aligned}
\operatorname{vor}_{0} & <-0.221, \\
\tau_{0} & >0.486 .
\end{aligned}
$$

3. If there are two flat quarters and no upright quarters, there is a quadrilateral subregion $F$. It satisfies

$$
\begin{aligned}
\operatorname{vor}_{0} & <-0.168, \\
\tau_{0} & >0.352 .
\end{aligned}
$$

These are twice the constants appearing in Inequality 11.

4. If there is an edge of length between $2 t_{0}$ and $2 \sqrt{2}$ running between two opposite corners of the hexagonal cluster, and if there are no flat or upright quarters on one side, leaving a quadrilateral region $F$, then $F$ satisfies

$$
\begin{aligned}
\operatorname{vor}_{0} & <-0.075, \\
\tau_{0} & >0.176 .
\end{aligned}
$$

5. If the hexagonal cluster has an upright diagonal with context $(4,2)$, and if there are no flat quarters (Fig. 25.5), then the hexagonal cluster $R$ satisfies

$$
\begin{aligned}
\sigma_{R} & <-0.297, \\
\tau_{R} & >0.504 .
\end{aligned}
$$

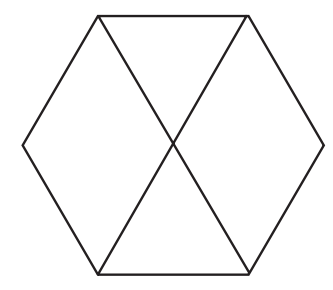

Fig. 25.5. A hexagonal cluster with context $(4,2)$. 




Fig. 25.6. A hexagonal cluster with context $(4,2)$.

6. If the hexagonal cluster has an upright diagonal with context $(4,2)$, and if there is one unmasked flat quarter (Fig. 25.6), let $\{F\}$ be the set of four subregions around the upright diagonal. (That is, take all subregions except for the flat quarter.) In the following inequality and Inequality 7, let $\sigma_{R}^{+}$be defined as $\sigma_{R}$ on quarters, and $\operatorname{vor}_{x}$ on other anchored simplices. $\tau_{R}^{+}$is the adapted squander function:

$$
\begin{aligned}
& \sum_{(4)} \sigma_{R}^{+}<-0.253, \\
& \sum_{(4)} \tau_{R}^{+}>0.4686 .
\end{aligned}
$$

7. If the hexagonal cluster has an upright diagonal with context $(4,2)$, and if there are two unmasked flat quarters (Fig. 25.7), let $\{F\}$ be the set of four subregions around the upright diagonal. (That is, take all subregions except for the flat quarters.)

$$
\begin{aligned}
& \sum_{(4)} \sigma_{R}^{+}<-0.2, \\
& \sum_{(4)} \tau_{R}^{+}>0.3992 .
\end{aligned}
$$

8. If the hexagonal cluster has an upright diagonal in context $(4,1)$, and if there are no flat quarters, let $\{F\}$ be the set of four subregions around the upright diagonal. Assume that the edge opposite the upright diagonal on the anchored simplex has length at least $2 \sqrt{2}$. (See Fig. 25.8.)

$$
\operatorname{vor}_{0, R}(D)+\sum_{(3)} \sigma(Q)<-0.2187
$$

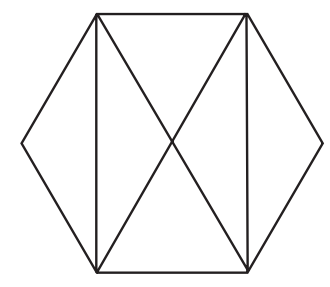

Fig. 25.7. A hexagonal cluster with context $(4,2)$. 


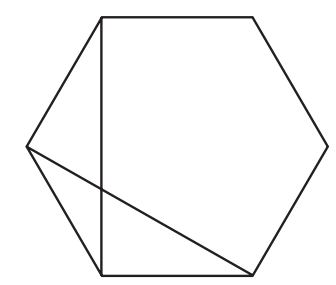

Fig. 25.8. A hexagonal cluster with context $(4,1)$.

$$
\tau_{0, R}(D)+\sum_{(3)} \tau(Q)>0.518
$$

9. In this same context, let $F$ be the pentagonal subregion along the upright diagonal. It satisfies

$$
\begin{aligned}
\operatorname{vor}_{0} & <-0.137 \\
\tau_{0} & >0.31
\end{aligned}
$$

10. If the hexagonal cluster has an upright diagonal in context $(4,1)$, and if there is one unmasked flat quarter, let $\{F\}$ be the set of four subregions around the upright diagonal. Assume that the edge opposite the upright diagonal on the anchored simplex has length at least $2 \sqrt{2}$. (There are five subregions, shown in Fig. 25.9.)

$$
\begin{gathered}
\operatorname{vor}_{0, R}(D)+\sum_{(3)} \sigma(Q)<-0.1657 \\
\tau_{0, R}(D)+\sum_{(3)} \tau(Q)>0.384
\end{gathered}
$$

11. In this same context, let $F$ be the quadrilateral subregion in Fig. 25.9. It satisfies

$$
\begin{aligned}
\text { vor }_{0} & <-0.084, \\
\tau_{0} & >0.176 .
\end{aligned}
$$

\subsubsection{Proof of the Inequalities}

Proposition 25.16. Inequalities 1-11 are valid.



Fig. 25.9. A hexagonal cluster with context $(4,1)$. 
We prove the inequalities in reverse order, $11-1$. The bounds ${ }^{144}$ vor $_{0}<0.009$ and $\tau_{0}>0.05925$ for flat quarters with diagonal $\sqrt{8}$ are used repeatedly. Some of the proofs make use of tcc-bounds, which are described in Section 12.9.

Proof of Inequalities 10 and 11 . Break the quadrilateral cluster into two simplices $S$ and $S^{\prime}$ along the long edge of the anchored simplex $S$. The anchored simplex $S$ satisfies $\tau(S) \geq 0, \sigma(S) \leq 0$. The other simplex satisfies $\tau_{0}\left(S^{\prime}\right)>0.176$ and $\operatorname{vor}_{0}\left(S^{\prime}\right)<$ -0.084 by an interval calculation. ${ }^{145}$ This gives Inequality 11 . For Inequality 10 , we combine these bounds with the linear programming bound on the four anchored simplices around the upright diagonal. From a series of inequalities ${ }^{146}$ we find that they score $<-0.0817$ and squander $>0.208$. Adding these to the bounds from Inequality 11 , we obtain Inequality 10.

Proof of Inequalities 8 and 9 . The pentagon is a union of an anchored simplex and a quadrilateral region. LP-bounds similar to those in the previous paragraph and based on the inequalities of Section 13.12 show that the loop scores at most -0.0817 and squanders at least 0.208 . If we show that the quadrilateral satisfies

$$
\begin{aligned}
\operatorname{vor}_{0} & <-0.137, \\
\tau_{0} & >0.31,
\end{aligned}
$$

then Inequalities 8 and 9 follow. If by deformations a diagonal of the quadrilateral drops to $2 \sqrt{2}$, then the result follows interval calculations. ${ }^{147}$ By this we may now assume that the quadrilateral has the form

$$
\left(a_{1}, 2, a_{2}, 2, a_{3}, 2, a_{4}, b_{4}\right), \quad a_{2}, a_{3} \in\left\{2,2 t_{0}\right\} .
$$

If the diagonals drop under 3.2 and $\max \left(a_{2}, a_{3}\right)=2 t_{0}$, again the result follows from interval calculations. ${ }^{148}$ If the diagonals drop under 3.2 and $a_{2}=a_{3}=2$, then the result follows from further interval calculations. ${ }^{149}$ So finally we attain by deformations $b_{4}=2 \sqrt{2}$ with both diagonals greater than 3.2. However, this does not exist, because

$$
\Delta\left(4,4,4,3.2^{2}, 4,8,3.2^{2}\right)<0 .
$$

Proof of Inequalities 5-7. Inequality 7 is derived in Section 13.12. Inequalities 5 and 6 are LP-bounds based on interval calculations. ${ }^{150}$

Proof of Inequality 4. Deform as in Section 12. If at any point a diagonal of the quadrilateral drops to $2 \sqrt{2}$, then the result follows from interval calculations ${ }^{151}$ and




Inequality 11:

$$
\begin{aligned}
\text { vor }_{0} & <0.009-0.084=-0.075 \\
\tau_{0} & >0+0.176=0.176 .
\end{aligned}
$$

Continue deformations until the quadrilateral has the form

$$
\left(a_{1}, 2, a_{2}, 2, a_{3}, 2, a_{4}, b_{4}\right), \quad a_{2}, a_{3} \in\left\{2,2 t_{0}\right\} .
$$

There is necessarily a diagonal of length $\leq 3.2$, because

$$
\Delta\left(4,4,3.2^{2}, 8,4,3.2^{2}\right)<0 .
$$

Suppose the diagonal between vertices $v_{2}$ and $v_{4}$ has length at most 3.2. If $a_{2}=2 t_{0}$ or $a_{3}=2 t_{0}$, the result follows from interval calculations ${ }^{152}$ and Inequality 11 . Take $a_{2}=a_{3}=2$. Inequality 4 now follows from interval calculations. ${ }^{153}$

Proof of Inequality 3. We prove that the quadrilateral satisfies

$$
\begin{aligned}
\operatorname{vor}_{0} & <-0.168, \\
\tau_{0} & >0.352 .
\end{aligned}
$$

There are two types of quadrilaterals: (a) two flat quarters whose central vertices are opposite corners of the hexagon, and (b) flat quarters who share a vertex. We consider case (a) first.

Case (a). We deform the quadrilateral as in Section 12.If at any point there is a diagonal of length at most 3.2, the result follows from Inequalities 10 and 11. Otherwise, the deformations give us a quadrilateral:

$$
\left(a_{1}, 2, a_{2}, 2 t_{0}, a_{3}, 2, a_{4}, 2\right), \quad a_{i} \in\left\{2,2 t_{0}\right\} .
$$

The tcc approximation now gives the result (see Section 12.10).

Case (b). Label the vertices of the quadrilateral $v_{1}, \ldots, v_{4}$, where $\left(v_{1}, v_{2}\right)$ and $\left(v_{1}, v_{4}\right)$ are the diagonals of the flat quarter. Again, we deform the quadrilateral. If at any point of the deformation, we find that $\left|v_{1}-v_{3}\right| \leq 3.2$, the result follows from Inequalities 10 and 11. If during the deformation $\left|v_{2}-v_{4}\right| \leq 2 \sqrt{2}$, the result follows from interval calculations. ${ }^{154}$ If the diagonal $\left(v_{2}, v_{4}\right)$ has length at least 3.2 throughout the deformation, we eventually obtain a quadrilateral of the form

$$
\left(a_{1}, 2 t_{0}, a_{2}, 2, a_{3}, 2, a_{4}, 2 t_{0}\right), \quad a_{i} \in\left\{2,2 t_{0}\right\} .
$$

However, this does not exist:

$$
\Delta\left(4,4,3.2^{2},\left(2 t_{0}\right)^{2},\left(2 t_{0}\right)^{2}, 3.2^{2}\right)<0 .
$$

We may assume that $\left|v_{2}-v_{4}\right| \in[2 \sqrt{2}, 3.2]$. The result now follows from interval calculations. ${ }^{155}$

\footnotetext{
152 CALC-148776243.

153 CALC-128523606.

154 CALC-148776243, CALC-315678695.

155 CALC-315678695
} 
Proof of Inequality 2. This case requires more effort. We show that

$$
\begin{aligned}
\operatorname{vor}_{0} & <-0.221, \\
\tau_{0} & >0.486 .
\end{aligned}
$$

Label the corners $\left(v_{1}, \ldots, v_{5}\right)$ cyclically with $\left(v_{1}, v_{5}\right)$ the diagonal of the flat quarter in the hexagonal cluster. We use the deformation theory of Section 12. The proof appears in steps (1)-(6).

(1) If during the deformations, $\left|v_{1}-v_{4}\right| \leq 3.2$ or $\left|v_{2}-v_{5}\right| \leq 3.2$, the result follows from the Inequalities in Section 25.13.2 and 11. We may assume this does not occur.

(2) If an edge $\left(v_{1}, v_{3}\right),\left(v_{2}, v_{4}\right)$, or $\left(v_{3}, v_{5}\right)$ drops to $2 \sqrt{2}$, continue with deformations that do not further decrease this diagonal. If $\left|v_{1}-v_{3}\right|=\left|v_{3}-v_{5}\right|=2 \sqrt{2}$, then the result follows from interval calculations. ${ }^{156}$

If we have $\left|v_{1}-v_{3}\right|=2 \sqrt{2}$, deform the figure to the form

$$
\left(a_{1}, 2, a_{2}, 2, a_{3}, 2, a_{4}, 2, a_{5}, 2 t_{0}\right), \quad a_{2}, a_{4}, a_{5} \in\left\{2,2 t_{0}\right\} .
$$

Once it is in this form, break the flat quarter $\left(0, v_{1}, v_{2}, v_{3}\right)$ from the cluster and deform $v_{3}$ until $a_{3} \in\left\{2,2 t_{0}\right\}$. The result follows from an interval calculation. ${ }^{157}$

We handle a boundary case of the preceding calculation separately. After breaking the flat quarter off, we have the cluster

$$
\left(a_{1}, 2 \sqrt{2}, a_{3}, 2, a_{4}, 2, a_{5}, 2 t_{0}\right), \quad a_{3}, a_{4}, a_{5} \in\left\{2,2 t_{0}\right\} .
$$

If $\left|v_{1}-v_{4}\right|=3.2$, we break the quadrilateral cluster into two pieces along this diagonal and use interval calculations ${ }^{158}$ to conclude the result. This completes the analysis of the case $\left|v_{1}-v_{3}\right|=2 \sqrt{2}$.

(3) If $\left|v_{2}-v_{4}\right| \leq 3.2$, then deform until the cluster has the form

$$
\left(a_{1}, 2, a_{2}, 2, a_{3}, 2, a_{4}, 2, a_{5}, 2 t_{0}\right), \quad a_{1}, a_{3}, a_{5} \in\left\{2,2 t_{0}\right\} .
$$

Then cut along the special simplex to produce a quadrilateral. Disregarding cases already treated by the interval calculations, ${ }^{159}$ we can deform it to

$$
\left(a_{1}, 2, a_{2}, 2 \sqrt{2}, a_{4}, 2, a_{5}, 2 t_{0}\right), \quad a_{i} \in\left\{2,2 t_{0}\right\},
$$

with diagonals at least 3.2. The result now follows from interval calculations. ${ }^{160}$

In summary of (1)-(3), we find that by disregarding cases already considered, we may deform the cluster into the form

$$
\begin{aligned}
& \left(a_{1}, 2, a_{2}, 2, a_{3}, 2, a_{4}, 2, a_{5}, 2 t_{0}\right), \quad a_{i} \in\left\{2,2 t_{0}\right\}, \\
& \left|v_{1}-v_{3}\right|>2 \sqrt{2},\left|v_{3}-v_{5}\right|>2 \sqrt{2},\left|v_{2}-v_{4}\right|>3.2 \text {. } \\
& 156 \text { CALC-148776243, CALC-673399623. } \\
& 157 \text { CALC-297256991. } \\
& 158 \text { CALC-861511432. } \\
& 159 \text { CALC-861511432. } \\
& 160 \text { CALC-746445726. }
\end{aligned}
$$


(4) Assume $\left|v_{1}-v_{3}\right|,\left|v_{3}-v_{5}\right| \leq 3$.2. If $\max \left(a_{1}, a_{3}, a_{5}\right)=2 t_{0}$, we invoke interval calculations ${ }^{161}$ to prove the inequalities. So we may assume $a_{1}=a_{3}=a_{5}=2$. The result now follows from interval calculations. ${ }^{162}$ This completes the case $\left|v_{1}-v_{3}\right|,\left|v_{3}-v_{5}\right| \leq$ 3.2 .

(5) Assume $\left|v_{1}-v_{3}\right|,\left|v_{3}-v_{5}\right| \geq 3.2$. We deform to

$$
\left(a_{1}, 2, a_{2}, 2, a_{3}, 2, a_{4}, 2, a_{5}, 2 t_{0}\right), \quad a_{i} \in\left\{2,2 t_{0}\right\} .
$$

If $a_{2}=2 t_{0}$ and $a_{1}=a_{3}=2$, then the simplex does not exist by Section 13.7. Similarly, $a_{4}=2 t_{0}, a_{5}=a_{3}=2$ does not exist. The tcc bound gives the result except when $a_{2}=a_{4}=2$. The condition $\left|v_{2}-v_{4}\right| \geq 3.2$ forces $a_{3}=2$. These remaining cases are treated with interval calculations. ${ }^{163}$

(6) Assume $\left|v_{1}-v_{3}\right| \leq 3.2$ and $\left|v_{3}-v_{5}\right| \geq 3.2$. This case follows from deformations and interval calculations. ${ }^{164}$ This completes the proof of Inequality 2.

Proof of Inequality 1. Label the corners of the hexagon $v_{1}, \ldots, v_{6}$. The proof to this inequality is similar to the other cases. We deform the cluster by the method of Section 12 until it breaks into pieces that are small enough to be estimated by interval calculations. If a diagonal between opposite corners has length at most 3.2 , then the hexagon breaks into two quadrilaterals and the result follows from Inequality 25.13.2.

If a flat quarter is formed during the course of deformation, then the result follows from Inequality 2 and interval calculations. ${ }^{165}$ Deform until the hexagon has the form

$$
\left(a_{1}, 2, a_{2}, 2, \ldots, a_{6}, 2\right), \quad a_{i} \in\left\{2,2 t_{0}\right\} .
$$

We may also assume that the hexagon is convex (see Section 12.12).

If there are no special simplices, we consider the tcc-bound. The tcc-bound implies Inequality 1 , except when $a_{i}=2$, for all $i$. However, if this occurs, the perimeter of the convex spherical polygon is $6 \operatorname{arc}(2,2,2)=2 \pi$. Thus, there is a pair of antipodal points on the hexagon. The hexagon degenerates to a lune with vertices at the antipodal points. This means that some of the angles of the hexagon are $\pi$. One of the tcc's has the form $C(2,1.6, \pi)$, in the notation of Section 12.10 . With this extra bit of information, the tcc bound implies Inequality 1 .

If there is one special simplex, say $\left|v_{5}-v_{1}\right| \in[2 \sqrt{2}, 3.2]$, we remove it. The score of the special simplex is ${ }^{166}$

$$
\begin{array}{llll}
\operatorname{vor}_{0}<0, & \tau_{0}>0.05925, & \text { if } & \max \left(\left|v_{1}\right|,\left|v_{5}\right|\right)=2 t_{0}, \\
\operatorname{vor}_{0}<0.0461, & \tau_{0}>0, & \text { if } & \left|v_{1}\right|=\left|v_{5}\right|=2,
\end{array}
$$

The resulting pentagon can be deformed. If by deformations, we obtain $\left|v_{2}-v_{5}\right|=$ 3.2 or $\left|v_{1}-v_{4}\right|=3.2$, the result follows from Inequalities 25.13.2 and two interval calculations. ${ }^{167}$

\footnotetext{
161 CALC-148776243, CALC-297256991.

162 CALC-897046482.

163 CALC-928952883.

164 CALC-297256991, CALC-673800906.

165 CALC-148776243.

166 CALC-148776243.

167 CALC-725257062, CALC-977272202.
} 
If $\left|v_{5}-v_{1}\right|=2 \sqrt{2}$, we use Inequality 2 and interval calculations ${ }^{168}$ unless $\left|v_{1}\right|=$ $\left|v_{5}\right|=2$. If $\left|v_{1}\right|=\left|v_{5}\right|=2$, we use interval calculations. ${ }^{169}$ If a second special simplex forms during the deformations, the result follows from interval calculations. ${ }^{170}$

The final case of Inequality 1 to consider is that of two special simplices. We divide this into two cases: (a) The central vertices of the specials are $v_{2}$ and $v_{6}$. (b) The central vertices are opposite $v_{1}$ and $v_{4}$. In case (a) the result follows by deformations and interval calculations. ${ }^{171}$ In case (b) the result follows by deformations and interval calculations. ${ }^{172}$ This completes the proof of Inequality 1 and the proof of the proposition.

\subsection{Conclusion}

By combinations of branching along the lines set forth in the preceding sections, a sequence of linear programs is obtained that establishes that $\sigma(D)$ is less than $8 p t$. For details of particular cases, the interested reader can consult the log files in [Ha16], which record which branches are followed for any given tame graph. (For most tame graphs, a single linear program suffices.)

This completes the proof of the Kepler conjecture.

\section{References}

[Ha6] T. C. Hales, Sphere packings, I, Discrete Comput. Geom. 17 (1997), 1-51.

[Ha14] T. C. Hales, Some algorithms arising in the proof of the Kepler conjecture, in Discrete and Computational Geometry, Algorithms and Combinatorics, vol. 25, Springer-Verlag, Berlin, 2003, pp. 489-507.

[Ha15] T. C. Hales, A proof of the Kepler conjecture, Ann. of Math. 162 (2005), 1065-1185.

[Ha16] T. C. Hales, Computer Resources for the Kepler Conjecture, http://annals.math.princeton.edu/ keplerconjecture/. (The source code, inequalities, and other computer data relating to the solution are also found at http://xxx.lanl.gov/abs/math/ 9811078v1.)

Received November 11, 1998, and in revised form September 12, 2003, and July 25, 2005.

Online publication February 27, 2006.

168 CALC-148776243.
169 CALC-377409251.
170 CALC-586214007.
171 CALC-89384104.
172 CALC-859726639. 